\title{
The interaction of fingermark deposits on metal surfaces and potential ways for visualisation
}

G. Wightman*, F. Emery, C. Austin, I. Andersson, L. Harcus, G. Arju, C. Steven

School of Science Engineering and Technology, University of Abertay, Bell Street, Dundee DD1 $1 \mathrm{HG}$, Scotland, United Kingdom

This is the accepted manuscript (C) 2015, Elsevier

Licensed under the Creative Commons Attribution-NonCommercial-NoDerivatives 4.0 International http://creativecommons.org/licenses/by-nc-nd/4.0/

(cc) BY-NC-ND

The published article is available from doi:

http://dx.doi.org/10.1016/j.forsciint.2015.01.035 


\section{Elsevier Editorial System(tm) for Forensic Science International Manuscript Draft}

Manuscript Number: FSI-D-14-00774R1

Title: The interaction of fingermark deposits on metal surfaces and potential ways for visualisation

Article Type: Original Research Article

Keywords: fingerprint; latent; oxidation; film

Corresponding Author: Dr. Graham Wightman,

Corresponding Author's Institution: University of Abertay

First Author: Graham Wightman

Order of Authors: Graham Wightman; F. Emery; C. Austin; I Andersson; L. Harcus; G. Arju; C. Steven

Abstract: The interaction of fingermark deposits on metals has been examined by a variety of techniques. Visualisation by film growth has been the main area of investigation through: thermal oxidation, anodising, peroxide solution, and the interaction with vapour of iodine and ammonium sulphide. Corrosion of the underlying metal has also been examined as an alternative means of visualisation.

Confocal microscopy was used to look at the film thickness and corrosion products around the prints. Scanning electron microscopy and energy dispersion of X-rays (SEM-EDX) examined a number of metal samples to investigate film growth and the elemental distribution. The observations suggest that differential oxidation was occurring as well as corrosion into the metal. Fingermark deposits on metals can corrode into the metal depending on the reactivity of the metal and leave a recoverable mark. However, fingermark deposits can also alter the rate of chemical reaction of the substrate metal by oxidation. In some cases organic matter can inhibit reaction, both when forming an oxide layer and when corroding the metal. However, signs of third level detail from pore contact may also be visible and the monovalent ions from salts could also influence film growth.

Whilst further work would need to be carried out to decide whether any of these techniques may have application in fingermark recovery, this study does suggest that fingermarks on metals may be recoverable after incidents such as fires or immersion in water. 
Acknowledgments

\section{Acknowledgement}

The loan of a confocal microscope (LEXT OLS4000) and an opto-digital microscope (DSX100) by Olympus, and EPSRC's support for access to the TEM/SEM facility at University of St Andrews are gratefully acknowledged 
School of Science Engineering and Technology,,

University of Abertay Dundee

Bell Street

Dundee

DD5 3JS

Scotland

10 September 2014

Dear Dr Margot,

Further to our correspondence, I would like to resubmit a paper for your journal, Forensic Science International. Although I disagree with the reviewers on a couple of points I have accepted their view and I believe I have accommodated all of the issues that they raised. As the reviewers said it was 'an interesting study of corrosion of metals by fingermark deposits' and it was 'a well thought out fundamental study' and 'a sensible and informative approach', I would be grateful if you could reconsider the revised paper..

The study looks at a variety of techniques for enhancement of fingermarks on metals by chemical reaction, and employed confocal microscopy and SEM/EDX to examine the reaction products. This has helped provide some insights into the mechanism of enhancement as well as suggesting areas for further study.

Yours sincerely,

Dr G Wightman 


\title{
The interaction of fingermark deposits on metal surfaces and potential ways for visualisation
}

\author{
G.Wightmana, F. Emery, C. Austin, I. Andersson, L. Harcus, G. Arju, C. Steven \\ a(Corresponding Author) \\ School of Science Engineering and Technology, \\ University of Abertay, \\ Bell Street, \\ Dundee, \\ DD1 1HG, \\ Scotland. \\ g.wightman@abertay.ac.uk
}

Telephone: +44 1382308659

\begin{abstract}
The interaction of fingermark deposits on metals has been examined by a variety of techniques. Visualisation by film growth has been the main area of investigation through: thermal oxidation, anodising, peroxide solution, and the interaction with vapour of iodine and ammonium sulphide. Corrosion of the underlying metal has also been examined as an alternative means of visualisation.

Confocal microscopy was used to look at the film thickness and corrosion products around the prints. Scanning electron microscopy and energy dispersion of X-rays (SEM-EDX) examined a number of metal samples to investigate film growth and the elemental distribution. The observations suggest that differential oxidation was occurring as well as corrosion into the metal. Fingermark deposits on metals can corrode into the metal depending on the reactivity of the metal and leave a recoverable mark. However, fingermark deposits can also alter the rate of chemical reaction of the substrate metal by oxidation. In some cases organic matter can inhibit reaction, both when forming an oxide layer and when corroding the metal. However,
\end{abstract}


signs of third level detail from pore contact may also be visible and the monovalent ions from salts could also influence film growth.

Whilst further work would need to be carried out to decide whether any of these techniques may have application in fingermark recovery, this study does suggest that fingermarks on metals may be recoverable after incidents such as fires or immersion in water. 


\section{Highlights}

- The potential development of latent fingermarks on metals has been examined by a variety of techniques.

- Thermal oxidation, anodising, reaction with iodine or ammonium sulphide vapour, and peroxide were all examined for film growth along with a study of the selective corrosion of the metal substrate.

- SEM, EDX and confocal microscopy have been used to identify how fingermark deposits can interact with the metal and reagent

- There is potential for fingermark recovery on metal after fires or immersion in water. 


\title{
The interaction of fingermark deposits on metal surfaces and potential ways for visualisation
}

\author{
Abstract \\ The interaction of fingermark deposits on metals has been examined by a variety \\ of techniques. Visualisation by film growth has been the main area of \\ investigation through: thermal oxidation, anodising, peroxide solution, and the \\ interaction with vapour of iodine and ammonium sulphide. Corrosion of the \\ underlying metal has also been examined as an alternative means of \\ visualisation. \\ Confocal microscopy was used to look at the film thickness and corrosion \\ products around the prints. Scanning electron microscopy and energy dispersion \\ of X-rays (SEM-EDX) examined a number of metal samples to investigate film \\ growth and the elemental distribution. The observations suggest that differential \\ oxidation was occurring as well as corrosion into the metal. Fingermark deposits \\ on metals can corrode into the metal depending on the reactivity of the metal and \\ leave a recoverable mark. However, fingermark deposits can also alter the rate \\ of chemical reaction of the substrate metal by oxidation. In some cases organic \\ matter can inhibit reaction, both when forming an oxide layer and when corroding \\ the metal. However, signs of third level detail from pore contact may also be \\ visible and the monovalent ions from salts could also influence film growth. \\ Whilst further work would need to be carried out to decide whether any of these \\ techniques may have application in fingermark recovery, this study does suggest \\ that fingermarks on metals may be recoverable after incidents such as fires or \\ immersion in water.
}




\section{Introduction}

Metals are widely used although often they are often coated for decorative purposes and corrosion protection. In the case of a fire scene the metallic objects may be the only artefacts remaining intact (e.g. petrol cans or car bodies). Similarly, firearms and cartridge cases as well as knife blades, metal railings, door handles and door push-plates are metallic objects that may be present at other crime scenes. Metal theft is also an increasing problem with war memorial plaques and copper cabling being added to lead roofing and silverware as targets for criminals. Historic artefacts in museums may also contain a latent record of who may have handled them. Whilst latent marks on metals may be developed by conventional techniques, it is important to understand the limits of recovery (for example, in a fire) and the potential for enhanced recovery.

Recent work by Williams et al [1, 2] and Bond [3-5] has increased the interest in this area. Williams used a scanning Kelvin microprobe to measure the potential across a surface film on 100 micron spacing and interpolated data with a commercial software package. Chloride ions in eccrine deposits reduced the passive oxide thickness, allowing a map of the latent mark to be produced. Fingermarks could be identified even after heating (as in a fire) or after being painted over. In Bond's initial studies latent fingermarks were heated by propane burner to simulate the effect of a fire, and enhancement was sometimes observed. Bond then studied the effect of storage time and humidity on mark development and found the degree of development was independent of both these variables over the range of conditions examined. Electrostatic enhancement was investigated, and development occurred due to the difference in resistivity of different regions of the fingermark affecting the powder adhesion. It was hypothesised that heat destroys the organic components present in the perspiration residue and then a heat-induced reaction occurs. Bond proposed that 'under certain conditions, the current density at the anode can result in the formation of a localized large concentration of metal ions that attract negatively 
charged ions and, for chloride ions this leads to the formation of hydrochloric acid in an autocatalytic reaction which corrodes the metal'.

Wightman and O' Connor [6] used heat to enhance latent fingermarks on metals, and in their study an electrically heated furnace was used instead of a flame in order to give greater control over the heating conditions. However, it was identified that enhancement was occurring through differential oxidation with regions of the fingermark oxidising at different rates. This resulted in interference colours and these colour differences were enhancing contrast. In the case of stainless steel a range of colours were observed but with brass contrast occurred as shades of light and dark due to absorption of part of the spectrum by the brass. Subsequent work by Bond [7] using X-ray photoelectron and Auger electron spectroscopy has confirmed the differential oxidation mechanism. There is forensic interest in recovering fingermarks off gun cartridges but high temperatures and abrasion in the weapon can destroy latent fingermarks and hence until recently firearms cartridge cases were not considered high priority for fingermark recovery but were examined primarily for tool marks. However, Bentsen et al [8] studied a range of techniques on spent cartridge cases and found that some fingermarks could be recovered. Dominick et al [9] demonstrated that latent marks could still be recovered after heating to $800{ }^{\circ} \mathrm{C}$, although their study used glass and ceramic rather than metals as a substrate. Gashi et al [10] have shown that temperatures during the discharge of firearms may be much lower than previously thought. In their study thermal imaging was carried out whilst a cartridge was being fired from a Browning pistol and the thermal profile at various time intervals during the firing were recorded using 8-9 $\mu \mathrm{m}$ radiation. Temperatures recorded were less than $450 \mathrm{~K}\left(180^{\circ} \mathrm{C}\right)$ due to the thermal mass of the cartridge and gun and they were able to recover alleles from fingermarks on the fired cartridges. These studies therefore suggest that fingermarks may sometimes be recoverable after firearms incidents or arson cases. 
A number of liquid phase techniques have been studied to enhance latent fingermarks on metals. Jasuja et al [11] used aqueous electrolytes (primarily acid and base solutions) to enhance fingermarks on metals and concluded that secretions act as a barrier for reaction with the metal. As with differential oxidation, the technique is dependent on the reaction with the substrate, not with the deposit and the deposit is acting as an inhibiting barrier. Beresford et al [12] used electrochromic deposition of polyaniline and poly 3, 4ethylenedioxythiophene which deposits between ridges in fingermarks, again suggesting an inhibiting effect. By controlling the oxidation state colour contrast is achieved and enhances observation. McKenzie et al [13] used electrochemical displacement in a molten salt as their preferred technique for reaction with the metal substrate and used copper or brass in a silver nitrate - choline chloride urea - ethylene glycol eutectic. A displacement reaction occurs with copper replacing the more electropositive ions in the molten salt electrolyte. Other displacement reactions have also been investigated, for example the work of Migron et al using palladium [14].

Both Cantu et al [15] and Leben and Ramotowski [16] report on the use of gunblue treatment of cartridge cases and the copper selenide deposit could potentially reveal latent marks. Again, reaction is occurring between the metal and the reagent to form a film, but residues from fingermarks inhibit deposition and these can be enhanced by controlled oxidation of the copper selenide film. According to McDermott [17], James has recently reported a patination process for recovering fingermarks on ammunition, although details are still to be published. A student project in Hong Kong in 2002-3 [18] examined the possibility of electrolysis for fingermark recovery and presented good recovery of fingermarks on reactive metals, but little else seems to have been reported on electrolysis apart from recent work by Nizam et al [19] recovering fingermarks on brass cartridge cases by electrolysis.

If it is postulated that different regions of the metal substrate can be oxidised at different rates and that the ridges of the fingermark are affecting growth of the oxide film, then control of the rate of oxidation may provide a means of improving 
enhancement. An alternative method for film growth on metals is the use of electrolysis or other oxidising agents such as iodine and ammonium sulphide which are known to produce interference fringes on copper [20] and may therefore present a non-thermal route to enhancement. The present study was therefore carried out in order to better understand how fingermarks affect chemical reaction of the metal substrate and to investigate other film growth methods. 


\section{Experimental}

\subsection{Oxidation: Thermal Enhancement}

Metal discs were cut to approximately $5 \mathrm{~cm}$ squares, and fingermarks were loaded from three donors after they had washed their hands followed by $30 \mathrm{~min}$ inactivity to allow eccrine sweat to predominate in the mark. Loaded discs were photographed then placed on a metal tray before being placed inside the preheated furnace. The heating cycle looked at the effect of varying times of heating since the amount of reaction depends on time as well as temperature. After $1 \mathrm{~min}$ the tray was removed, allowed to cool, photographs were taken, and the tray returned to the furnace for a further $3 \mathrm{~min}$. This was repeated with 5, 7 and $9 \mathrm{~min}$ intervals. Three temperatures were employed for each metal, and four metals were investigated: brass, stainless steel, mild steel and copper (table 1) and operating conditions are given in table 2. Marks were graded on a 0-4 scale [21]. Poor enhancement occurred on $6 \mathrm{~mm}$ mild steel and a short series of tests was carried out on $0.5 \mathrm{~mm}$ sheet mild steel to investigate whether heat transfer was an issue and samples were heated at $400{ }^{\circ} \mathrm{C}$ and $600{ }^{\circ} \mathrm{C}$ for a 5 min period. Samples of each metal were also tested with different methods of support (on a metal tray or raised on a ceramic boat) in case heat transfer or air flow was an issue. Finally, virgin metal samples were heated with a flame and produced interference colours confirming that the materials used were oxidisable.

A selection of the marks was examined by scanning electron microscope for microstructure and microanalysis by EDX (energy dispersion of X-rays) to identify the reaction products.

\subsection{Alternative Oxidants}

Copper and brass were chosen for this series of tests since copper is known to react well with ammonium sulphide and with iodine [20], although a number of additional trials were also carried out on mild steel and stainless steel. In the case of oxidation by air the variables used were time and temperature for reaction but in the case of the sulphide and iodide films the variable was 
concentration. For ammonium sulphide trials three donors were again used and metal tokens were cleaned with acetone and loaded with eccrine fingermark deposits. The disc was placed in a petri dish in a fume hood and a drop of ammonium sulphide solution was placed in the dish and a lid covered the dish to prevent drafts and to minimise the smell as ammonium sulphide dissociates to yield hydrogen sulphide. The concentration was varied by using different dilutions of solution. In the case of iodine a similar procedure was employed with a crystal of iodine replacing the ammonium sulphide and concentration was varied by altering the iodine vapour pressure by warming the petri dish since vapour pressure depends exponentially on temperature according to the Clausius Clapeyron equation. Test conditions are given in table 3.

Peroxide solution can oxidise various materials and tooth whiteners are based on carbamide peroxide so the potential of these materials to form an oxide layer was investigated. Two dilutions of $\mathrm{H}_{2} \mathrm{O}_{2}(15 \%$ and $30 \%)$ for various time periods (1.5 $\min$ to $3 \mathrm{~h}$ ) were investigated to try and produce an oxide film. Carbamide peroxide solution was made up (12 $\mathrm{g}$ in $100 \mathrm{~mL}$ ) and the metal samples were placed in solution for varying periods of time.

A final technique studied for film growth was anodising. For this study 3 different metals that form protective oxides were used (aluminium, stainless steel, and tincoated steel) with 6 different anodising solutions under various direct current anodising conditions (table 4).

\subsection{Corrosion of the metal substrate}

An alternative approach to building up an oxide layer on the metal is to selectively remove the underlying metal. Acidified peroxide solution $(2.5 \mathrm{~mL}$ of 10 $\mathrm{M} \mathrm{HCl}$ to $40 \mathrm{~mL}$ of $30 \% \mathrm{H}_{2} \mathrm{O}_{2}$ and $26 \mathrm{~mL}$ of distilled water), and Fry's reagent (22.5 $\mathrm{g} \mathrm{CuCl}_{2}, 30 \mathrm{ml}$ of $36 \% \mathrm{HCl}$ and $25 \mathrm{~mL}$ distilled water) diluted with water were used. Metal samples that had been heated to form an oxide film were placed in solution for periods from $30 \mathrm{~s}$ to $5 \mathrm{~min}$. 
In two separate series of experiments samples of anodised tinned steel were treated with SPR suspension in water and selective corrosion was observed. Loaded mild steel discs were also placed in water or salt water for up to 28 days and were washed and observed.

\subsection{Examination of the Oxide Film}

Fingermarks were recorded photographically and examined with a Leica macroscope as well as being assigned a grade on a 0-4 scale. A selected sample of fingermarks was examined by SEM and EDX. In addition a number of fingermark films were examined by confocal microscope (Olympus LEXT OLS4000) and opto-digital microscope (Olympus DSX100).

In the case of aluminium, copper and brass, attempts were made to remove the oxide layer for examination. Aluminium oxide can be removed by amalgamation of the aluminium using mercury chloride solution. Copper forms a thick brittle oxide film that can fragment due to the different thermal expansion coefficients of oxide and metal and the oxide film could often be removed by applying a piece of forensic lifting tape. 
3 Results and Discussion

\subsection{Thermal oxidation}

The initial hypothesis had been that enhancement could be achieved through controlled oxidation by varying the length of time exposed to heat. However results were poorer than expected (table 5). Very seldom were grade 3 or grade 4 marks obtained, although a few were observed, mainly on brass. Some variation in recovery between marks from different donors was noted.

With fingermarks on copper poor enhancement was achieved, except for 2 donors at $600{ }^{\circ} \mathrm{C}$. The fingermark grade generally did not improve with extended periods of heating, although the mark did not degrade on extended heating. Temperature appeared to be a more important factor than time in developing fingermarks. The black copper oxide produced good contrast on the orange colour of the metal but one problem with copper was the brittle nature of its oxide which began to flake off as the metal contracted, thus appearing to make the enhanced mark fragile.

With brass two donors' fingermarks gave reasonable recovery when heated above $400{ }^{\circ} \mathrm{C}$ for periods over 5 to 10 minutes cumulative time (i.e. after the 3 or 5 min period) and the marks did not degrade with extended heating.

Stainless steel generally gave poor enhancement and an uneven oxide film formed. Time of heating had no apparent effect except at $750^{\circ} \mathrm{C}$ where the mark developed when heated for 4 min.

$6 \mathrm{~mm}$ thick mild steel gave poor recovery of latent marks. $0.5 \mathrm{~mm}$ mild steel underwent 'blueing' at $400{ }^{\circ} \mathrm{C}$ with no fingermark visible but at $600{ }^{\circ} \mathrm{C}$ no blueing was visible and an orange fingermark had appeared due to interference colours from the oxide film.

The series of tests carried out to compare the method of support showed no obvious trend. Again, these observations tend to suggest that temperature rather than time or air flow seems to be the more important factor.

3.2 Examination of the oxide film 
Samples were examined by scanning electron microscopy and EDX as well as confocal microscopy to study the nature of the oxide film.

Brass results are shown in figures 1-4 and table 6 . Figure 1 shows the fingermark as seen under the confocal microscope. This technique allowed a greater depth of focus and enabled a large area to be covered at higher magnification by stitching sequential images together (figure 1b). Black spots could be seen that were thought to correspond to pores and these were examined in 3D mode and the z-axis was expanded (figure 2). As can be seen, these dark regions which had originally been thought to be pits were actually standing proud of the metal by around 1 micron and the appearance is more like a crater with an annulus of higher material. Brass treated with salt solution in the same manner showed the same effect (figure 3) where there is also a depression into the metal surface and it is presumed that sodium chloride is causing corrosion into the metal and corrosion products are surrounding the corrosion site, as seen in the crosssectional profile.

SEM/EDX analysis was carried out and is shown in figure 4. It appears that the fingermark consists of an oxide layer of differing thicknesses and the EDX elemental map shows a higher sodium level as well as higher oxygen and lower copper in these regions (zinc may be higher but is inconclusive. and requires a longer scan time). Spot analysis by EDX of the ridges and hollows is given in table 6 , but due to the variation in analyses it was not possible to confirm whether zinc was preferentially oxidised. The interaction zone of the electron beam is also likely to affect the ratio of elements by producing $x$-rays from the underlying metal. However, there does appear to be a higher proportion of oxygen in the ridge region and it may be that sodium is enhancing oxidation rate due to being monovalent and introducing vacancies into the oxide structure.

Fingermarks could be enhanced on stainless steel and mild steel under some conditions of heating but SEM/EDX and confocal microscopy did not provide sufficient information to elicit a mechanism. Figure 5a shows a confocal micrograph of one example on mild steel and the oxide has built up along the 
ridges with darker spots that may correspond to pores. There appears to be dendritic growth occurring from the ridges and across the hollows which may suggest that the presence of residues left by ridge contact is enhancing oxidation in this case. Figure $5 b$ shows a stitched series of $3 D$ images with enhanced $z-$ axis showing that there is a difference in oxide thickness between ridges and hollows. Figure $5 \mathrm{c}$ is a similar $3 \mathrm{D}$ image of a pore showing the crater appearance.

Copper results are shown in figures 6-9. Copper formed a black oxide layer that could flake off to reveal underlying metal, although sometimes the oxide would split into two layers as seen in figure 6 with black $\mathrm{CuO}$, red $\mathrm{Cu}_{2} \mathrm{O}$ and copper metal. Confocal microscopy suggests that oxide preferentially fractures where there has been pore contact as spots can sometimes be seen on the surface of the oxide and can also be seen when the oxide flaked off (figure 7a). 3D images with an expanded $z$-axis are shown in figures $7 b$ and $7 c$ where the oxide can be seen and measured. The cross sectional profile shows a non-uniform oxide surface, possibly as part of the oxide film starts to break away and tilt. However, the oxide layer was about $5 \mu \mathrm{m}$ thick. The image also shows depressions in the copper metal, possibly due to pores, and in figure 7c a slight rise (about $0.5 \mu \mathrm{m}$ ) can be seen for a region on the oxide film which again may correspond to a pore. SEM and EDX analysis was carried out and figure 8 shows the SEM image where spots (presumed to be from pores) can be seen on the oxide film corresponding with ridges, and some fragmentation of oxide has occurred. EDX analysis simply confirmed the presence of copper oxide with no other elements detected (ie sodium was not identified in the oxide layer).

Whilst sometimes the fingermark could be seen in the oxide, in other cases no fingermark was observed on the oxide. However, evidence of a residual mark etched into the metal could be apparent when the oxide flaked off. An attempt was therefore made to lift the oxide film using lifting tape and the result can be seen in figures $9 \mathrm{a}$ and $9 \mathrm{~b}$ where there is a faint appearance of a fingermark through the oxide and the underlying mark was exposed after the lift. Often the fingermark caused the oxide to remain attached to the metal rather than spalling 
off (figure 9c). There was also some evidence of the mark in the lifted oxide layer (figure 10a) and so the same technique was also applied to brass (figure 10b) where oxide has been lifted and reveals some of the features of the mark. Lifting the oxide layer from aluminium using mercury chloride solution was tried as it undergoes a displacement reaction with aluminium to form mercury which then amalgamates with the aluminium to release the oxide layer. This would not be a technique for general application but from a research perspective it would allow more detailed analysis of the oxide film. However, the oxide layer was fragile and disintegrated.

Further work is required on removal of oxide layers but these observations suggest that recovery of fingermarks may be possible from metal artefacts of copper and brass after a fire.

3.3 Film formation without heating

\subsubsection{Ammonium sulphide}

An alternative way to produce a film and hence to potentially enhance the fingermark is by using another oxidant. A 100/1 dilution of ammonium sulphide solution produced too little vapour and no reaction was observed. However, at 10/1 dilution, enhancement could be achieved and results are shown in table 7 . Copper reacts very quickly and whilst the marks were temporarily enhanced, they very quickly degraded and this would be consistent with the mark inhibiting oxidation of the metal but eventually the film becomes so thick that contrast is not observed. On brass the marks appeared more slowly and were of a poorer grade but they did not fade, presumably due to a slower reaction rate. Figure 11 shows an example of enhancement by ammonium sulphide with a high level of corrosion in one corner close to the solution. After storage the marks did degrade due to further reaction with reagent still present on the metal. The use of ammonium sulphide is unlikely to present a viable method of enhancement but may help in understanding the mechanism of enhancement.

\subsection{2 lodine}


lodine reacts more slowly as it has a much lower vapour pressure. To increase the vapour pressure the petri dish was warmed in an oven at controlled temperature and periodically observed. Results obtained at 30,40 and $50{ }^{\circ} \mathrm{C}$ are given in table 8. As with the tests on differential oxidation with air, there was some variation in quality of the mark due to the donor, but iodine vapour appears to react at a controlled rate with both copper and brass and to enhance the mark. In general a longer period enhances the mark and no decline was observed with periods up to $8 \mathrm{~min}$. Brass appeared to give better results than copper.

SEM/EDX analysis confirmed the presence of copper and iodine and the ridge details. At higher magnification it appeared to be dendritic growth, although there also appears to be preferential growth in certain directions (figure 12b).

\subsubsection{Peroxide solution}

Hydrogen peroxide and carbamide solutions were tried with several metals (copper, brass, mild steel and stainless steel). The techniques produced a discolouration of the copper alloys similar to tarnishing, and the presence of fingermarks could be revealed, although over-oxidation could occur and destroy the mark. Bubbles were seen to form and these sometimes followed the detail of the fingermark (figure 13).

In the case of copper the metal darkens as the oxide layer forms but the ridges of the fingermark remain the original copper colour and this could be related to why the bubbles form in the shape of ridges. If the bubbles were disturbed they were dispersed and this prevented the fingermark from developing. Marks developed in 5-15 min with 15\% peroxide. Using 30\% peroxide copper gave visible marks after only $1.5 \mathrm{~min}$, although samples could remain in the solution for up to $5 \mathrm{~min}$ and the marks would still be very clearly visible.

Similar observations were made with brass: the metal oxidised around the mark and between the ridges so the brass went very dark and the ridges appeared silver. $15 \mathrm{~min}$ was found to be the best time for visualising fingermarks on brass with $15 \%$ solution but with $30 \%$ peroxide very few ridges were visible. This could be because of over - oxidation as the metal went very dark. 
Mild steel and stainless steel did not reveal any marks with hydrogen peroxide at $15 \%$ or $30 \%$.

With carbamide peroxide solution the first copper sample only had a sebaceousloaded mark on it and after 15 min the copper had darkened because of oxidation but the fingermark had remained light. On removal from the solution all of the solution on the mark had been repelled so the area was dry. This could be a hydrophobic effect resulting in oxidation of the gaps between the ridges but not the ridges themselves since these appeared the original colour of the copper. The second sample had both a sebaceous mark and an eccrine. Again the sebaceous mark acted in the same way as the first sample but the eccrine mark appeared to oxidise slightly and was darker than the original copper colour, but not as dark as the oxidised background, therefore the mark was visible but there was not a lot of contrast.

Three fingermarks on brass were analysed with different exposure times. The first was immersed for $5 \mathrm{~min}$ and there was a dark border around the sebaceous mark but no development of the ridges. The eccrine mark was visible as the ridges had not oxidised so they were lighter against a dark background. The second sample was in the solution for $10 \mathrm{~min}$, resulting in the sample going much darker but the sebaceous mark remaining golden. The eccrine mark was also light against the dark background; however the ridges had started to oxidise, but at a different rate to the rest of the oxide layer thus allowing it to be visible. The final sample was in for $15 \mathrm{~min}$ and the sebaceous mark ridges had darkened slightly so they were clearly visible as the mark was much lighter than the background. The eccrine mark also had a good contrast and was clearly visible. Peroxide may offer a means of recovery in some cases but is unlikely to be of general use.

\subsubsection{Anodising}

Anodising is another technique for controlled oxidation to form a film. A calculation of the charge transfer during electrolysis suggests that an oxide film comparable with wavelengths of light can be developed in a few minutes. In the present study 9 and $12 \mathrm{~V}$ were used with a current of $0.05-0.5 \mathrm{~A}$ for $5 \mathrm{~min}$ and 
maximum oxide thickness would be $0.8-10 \mu \mathrm{m}$, assuming $100 \%$ efficiency. In practice electrolysis incurs energy losses so these conditions were theoretically the correct order of magnitude to achieve interference colours. Several anodising acids were investigated with aluminium, stainless steel, and tinned steel but in practice only tinned steel achieved much enhancement (figure 14a). This is consistent with the findings of Anon [18] and Nazim [19] who used electrolysis on more reactive metals such as zinc and brass. It is slightly surprising that anodising was unsuccessful as it is known in the anodising industry that fingermarks can produce defects in the anodising of goods, and anodising is used artistically and commercially to produce interference colours [22]. One possible explanation is the high resistance of the protective oxide film due to the low vacancy defects and hence higher potential or longer time may be required for these passive metals. The use of anodising dye post-electrolysis did show some potential on anodised aluminium and needs further study (figure 14b).

\subsection{Corrosion of metal substrate}

Differential film growth could occur by two mechanisms: enhancement of growth by the salt present and is more likely to occur with eccrine deposits or a reduction in rate by an organic barrier which is more likely to occur with sebaceous deposits or from contaminated hands (e.g. greasy hands). For example, thermal oxidation may be enhanced by salt whereas peroxide oxidation may be inhibited by grease etc. It is therefore possible that selective dissolution of the substrate could also be achieved by similar mechanisms - corrosion by eccrine sweat or inhibition by sebaceous sweat. This was tested with acidified hydrogen peroxide, diluted Fry's reagent, salt water and fresh water on both untreated and oxidised fingermarks.

With acidified hydrogen peroxide, copper gave good results with clear marks after $40 \mathrm{~s}$. The surface of the copper reacted very quickly to form a dark colour, including between the ridges. The ridges themselves, however, retained the original shiny copper colour. The organic components of the marks may therefore be preventing the metal from oxidising by having a hydrophobic affect 
and repelling the solution. Brass was also treated for $40 \mathrm{~s}$ and it too became highly oxidised but the ridges of the mark remained the golden colour of brass. The marks had again prevented oxidation allowing the mark to be clearly visible. No fingermarks were visualised on the stainless steel and this will be because of the chromium oxide protecting it. Mild steel oxidised very quickly in the acidified solution, even after $3 \mathrm{~s}$ the fingermark had been obscured by the corrosion product. A fingermark, however, was developed after $2 \mathrm{~s}$ in the solution and although metal was still much oxidised a silver mark was visible where the ridges had not yet oxidised. This, however, is not suitable because there is a fine line between visualising a mark and over-oxidising the sample. One option to avoid this may be by using a less concentrated acid in the reagent.

Undiluted Fry's reagent rapidly corroded the metal (brass, tinned steel, and stainless steel) and erased the mark so a series of dilutions were carried out. Table 9 gives the results for a 10 -fold dilution with immersion periods from $30 \mathrm{~s}$ to $5 \mathrm{~min}$. The overall increase of the identifiable marks rose from $34 \%$ to $54 \%$. Etching may therefore offer some potential to enhance recovery.

It was observed that anodised tinned steel treated with water developed rust between ridges (figure 15). In an alternative study examining recovery of fingermarks from metals immersed in fresh and sea water it was noticed that a layer of rust formed over the metal plate but when the loosely adhering rust was rinsed off the underlying metal had preferential corrosion between the ridges. These observations are in agreement with each other although the metal was probably being protected by an oxide layer in one case and by organic secretions in the other.

From a practical point of view selective dissolution of the substrate would be a very last resort as it is destructive, but these observations do indicate how fingermarks on metals may subsequently be found, particularly where moisture or other atmospheric components cause corrosion or film formation. 


\section{Conclusion}

This study has examined a wide range of techniques to try and recover latent fingermarks on metals: thermal oxidation; anodising; oxidation by iodine and ammonium sulphide; aqueous oxidation by peroxide; and corrosion with acid and with water. During the study confocal microscopy and SEM/EDX have been used to try and understand the mechanism of fingermark development. The mechanism will vary according to the thermodynamic properties of the metal or the components of the alloy. However, the deposition of salts and organic compounds onto the metal is important. Deposits may inhibit or enhance reaction and cause differential oxidation. Salts may enhance the oxide growth by introducing defects into the oxide structure, but they can also result in corrosion into the metal substrate. Thus a fingermark may be observed both in the oxide film and in the underlying metal and this can have implications for the recovery of fingermarks on metallic objects recovered from fires. Likewise, differential corrosion may result in fingermarks being recovered from metallic objects buried in water or damp soil. 


\section{References}

[1] G. Williams, H. N. McMurray, D. A. Worsley, Latent fingerprint detection using a scanning Kelvin microprobe', J Forensic Sci., 2001, 46(5), 1085-1092. [2] G. Williams, N. McMurray, Latent fingerprint visualisation using a scanning Kelvin probe, Forensic Science International, 2007, 167, 102-9 .

[3] J. W. Bond, Visualisation of latent fingerprint corrosion of metallic surfaces, J. Forensic Sci., 2008, vol. 53(4), 812-822.

[4] J. W. Bond, The thermodynamics of latent fingerprint corrosion of metal elements and alloys, J. Forensic Sci., 2008, 53(6), 1344-1352.

[5] J. W. Bond On the electrical characteristics of latent finger mark corrosion of brass, J. Phys. D: Appl. Phys., 2008, 41, 125502 (10pp).

[6] G. Wightman, and D. O'Connor, The thermal visualisation of latent fingermarks on metallic surfaces, Forensic Science International., 2011, 204, 8896.

[7] J. W. Bond Optical enhancement of fingerprint deposits on brass using digital colour mapping, J Forensic. Sci., 2011, 56(5), 1285-1288.

[8] R. K. Bentsen, J. K. Brown, A. Dinsmore, K. K. Harvey, T. G. Kee, Post Firing Visualisation of Fingerprints on Spent Cartridge Cases, Science and Justice, 1996, 36, 3-8.

[9] A. J. Dominick, K. A. Laing, A comparison of six fingerprint enhancement techniques for the recovery of latent fingerprints from unfired cartridge cases, Journal of Forensic Identification, 2011, 61(2), 155-165.

[10] B. Gashi, M. R. Edwards, P. A. Sermon A. L. Courtney, D. Harrison, and Y. $\mathrm{Xu}$, Measurement of $9 \mathrm{~mm}$ cartridge case external temperatures and its forensic application, Forensic Science International 2010, 200, 21-27.

[11] O. M. Jasuja, G. Singh, and J. Almog, Development of latent fingermarks by aqueous electrolytes, Forensic Science International, 2011, 207, 215-222.

[12] A. L. Beresford, R. M. Brown, and R. A. Hillman, Electrochemical polymerisation of polyaniline and PEDOT (poly 3, 4 ethylenedioxythiophene), The seventh FORREST Conference, Nottingham Trent University, 29th - 30th June 2011. 
[13] K. J. McKenzie, R. A. Hillman, A. J. Goddard, S. Ensor, and A. Prodan, Electroless deposition of silver, The seventh FORREST Conference, Nottingham Trent University, 29th - 30th June 2011.

[14] Y. Migron, G. Hocherman, E. Springer, J. Almog, and D. Mandler, Visualisation of sebaceous fingerprints on fired cartridge cases: a laboratory study, J. For. Sci. 1997, 42(6), 986-992.

[15] A. A. Cantu, D. A. Leben, R. Ramotowski, J. Kopera and J. R. Simms, The use of acidified hydrogen peroxide to remove excess gun blue from gun bluetreated cartridge cases and to develop latent prints von untreated cartridge cases, J. For. Sci. 1998, 4 (2), 294-298.

[16] D. A. Leben and R. S. Ramotowski, Evaluation of Gun Blueing Solutions and their Ability to develop Latent Fingerprints on Cartridge Casings, J. Forensic Sci., 1998, 43(2), 294-298.

[17] J. McDermott, Fingerprint technique heralds new investigation era, Police Oracle, 7 February 2014, available on http://www.policeoracle.com/news/Investigation/2014/Feb/07/Fingerprinttechnique-heralds-new-investigation-era 78160.html, accessed 17 June 2014. [18] Anon, Holy Family Canossian College, Fingerprint Detective - Electrolysis, Finalist in the $8^{\text {th }}$ Hong Kong Chemistry Olympiads for Secondary Schools (2002-2003): available on

http://resources.edb.gov.hk/ science/hkcho/8s/Canossian.pdf, accessed 2 May 2014.

[19] F. Nizam, W. Knaap, and J. D. Stewart, Development of Fingerprints Using Electrolysis: A Technical Report Into the Development of Fingerprints on Fired Brass Cartridge Cases, Journal of Forensic Identification, 2012, 62( 2), 129-142. [20] J. P. Chilton, Principles of metallic corrosion, Royal Institute of Chemistry Monographs for teachers, 1964.

[21] Sears V G, Bleay S M, Bandey H L, Bowman V J, A methodology for finger mark research, Sci Justice. 2012, 52(3), 145-160.

[22] L. Bartlett, An unusual phenomenon observed when anodising CP titanium to produce coloured surfaces for jewellery and other decorative uses, Optics \& 
Laser Technology 2006, 38, 440-444. 
Table 1. Metal samples used

\begin{tabular}{llll} 
Metal & Specification & Typical Analysis & Thickness mm \\
\hline Aluminium & BS1470 SIC (1987) & $>99 \% \mathrm{Al}$ & 1.1 \\
Brass & BS2874/CZ121M & $58 \% \mathrm{Cu}, 39 \% \mathrm{Zn}, 3 \% \mathrm{~Pb}$ & 0.8 or 0.3 \\
Copper & BS2874/C101 & $99.9 \% \mathrm{Cu}$ & 0.35 \\
Stainless & BS1449 pt 2304S31 & $17.5-19.0 \% \mathrm{Cr}, 8.0-11.0 \%$ & 1.0 \\
steel & & $\mathrm{Ni}$ & \\
Mild steel & AISI-1008/AISI-1010 & $99.2-99.6 \% \mathrm{Fe}, 0.3-0.6 \%$ & 6.0 or 0.5 \\
& & Mn, 0.08-0.13 \% C & \\
Tinned steel & BS 1449 Part 1 CR4 & $6 \mu \mathrm{m} \mathrm{99.98 \%} \mathrm{Sn}$ & 0.3
\end{tabular}


Table 2. Test conditions for metal oxidation.

\begin{tabular}{lll} 
Metal & $\begin{array}{l}\text { Temperature } \\
{ }^{\circ} \mathrm{C}\end{array}$ & Times min \\
\hline Brass & $200,400,600$ & $1,3,5,7,9$ \\
Copper & $200,400,600$ & $1,3,5,7,9$ \\
$\begin{array}{l}\text { Stainless } \\
\text { steel }\end{array}$ & $600,750,900$ & $1,3,5,7,9$ \\
Mild steel & $450,650,850$ & $1,3,5,7,9$
\end{tabular}


Table 3. Test conditions for sulphide and iodide reaction.

$$
\text { Saturated ammonium lodine temperature }
$$

Metal sulphide dilution $\quad{ }^{\circ} \mathrm{C}$

Brass $\quad 1,0.1,0.01 \quad 30,40,50$

Copper $\quad 1,0.1,0.01 \quad 30,40,50$ 
Table 4.Test conditions for anodising

Anodising solution

\begin{tabular}{|l|l|l|l|l|l|}
\hline $\begin{array}{l}\text { Sulphuric } \\
\text { acid }\end{array}$ & $\begin{array}{l}\text { Chromic } \\
\text { acid }\end{array}$ & $\begin{array}{l}\text { Phosphoric } \\
\text { acid }\end{array}$ & $\begin{array}{l}\text { Sulfosalicylic } \\
\text { acid }\end{array}$ & $\begin{array}{l}\text { Tartaric } \\
\text { acid }\end{array}$ & Boric acid \\
\hline
\end{tabular}

Anodising conditions

$\begin{array}{rrl}9 \mathrm{~V} & 0.05 \mathrm{~A} & 5 \mathrm{~min} \\ 12 \mathrm{~V} & 0.05 \mathrm{~A} & 5 \mathrm{~min} \\ 9 \mathrm{~V} & 0.1 \mathrm{~A} & 5 \mathrm{~min} \\ 12 \mathrm{~V} & 0.1 \mathrm{~A} & 5 \mathrm{~min} \\ 9 \mathrm{~V} & 0.5 \mathrm{~A} & 5 \mathrm{~min} \\ 12 \mathrm{~V} & 0.5 \mathrm{~A} & 5 \mathrm{~min} \\ 12 \mathrm{~V} & 1 \mathrm{~A} & 15 \mathrm{~min}\end{array}$




\section{Table 5Thermal enhancement of fingermarks on metal}

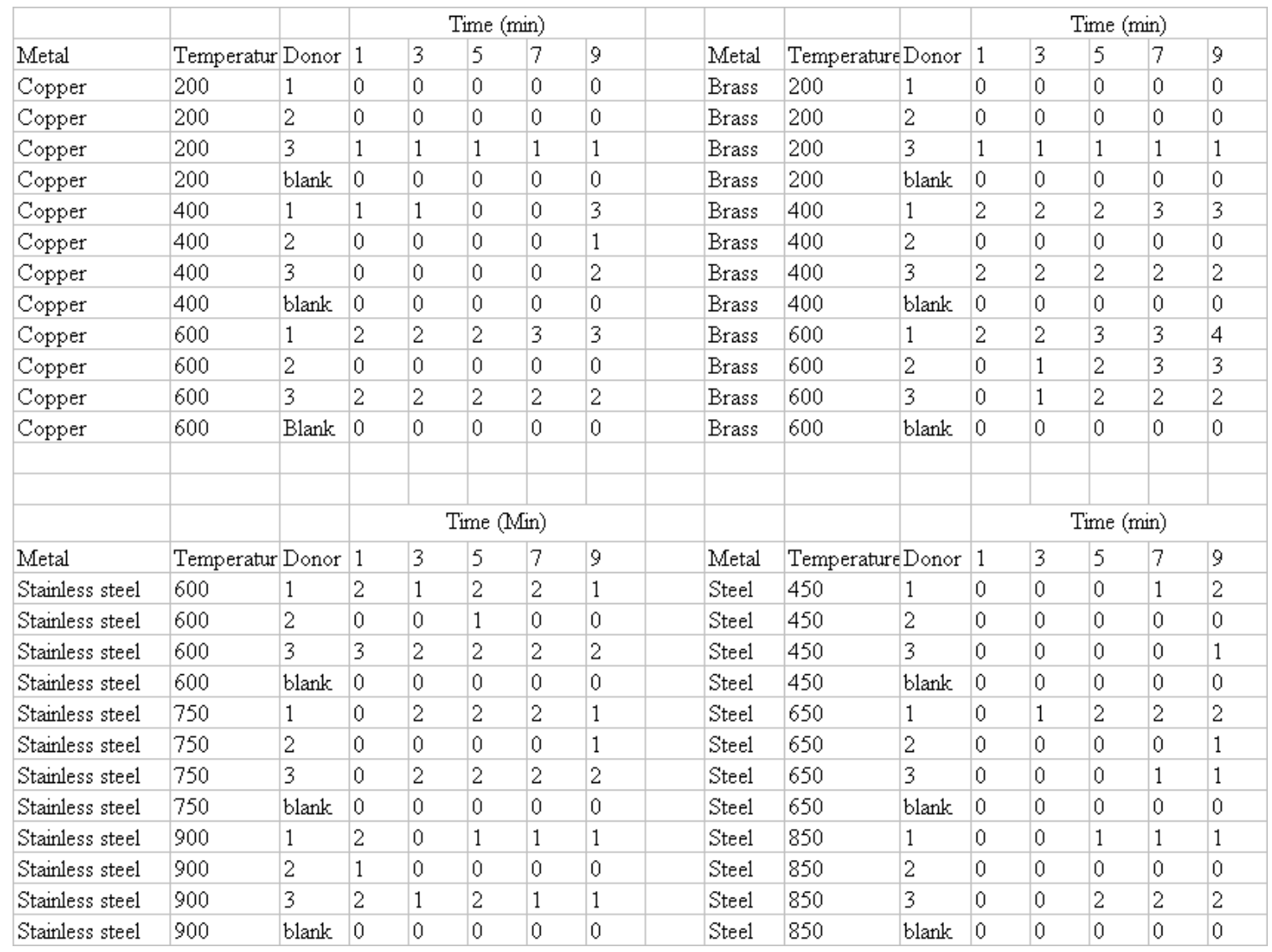


Time (min)

$\begin{array}{llllllll}\text { Metal } & \text { Temperature } & \text { Donor } & 1 & 3 & 5 & 7 & 9 \\ \text { Copper } & 200 & 1 & 0 & 0 & 0 & 0 & 0 \\ \text { Copper } & 200 & 2 & 0 & 0 & 0 & 0 & 0 \\ \text { Copper } & 200 & 3 & 1 & 1 & 1 & 1 & 1 \\ \text { Copper } & 200 & \text { blank } & 0 & 0 & 0 & 0 & 0 \\ \text { Copper } & 400 & 1 & 1 & 1 & 0 & 0 & 3 \\ \text { Copper } & 400 & 2 & 0 & 0 & 0 & 0 & 1 \\ \text { Copper } & 400 & 3 & 0 & 0 & 0 & 0 & 2 \\ \text { Copper } & 400 & \text { blank } & 0 & 0 & 0 & 0 & 0 \\ \text { Copper } & 600 & 1 & 2 & 2 & 2 & 3 & 3 \\ \text { Copper } & 600 & 2 & 0 & 0 & 0 & 0 & 0 \\ \text { Copper } & 600 & 3 & 2 & 2 & 2 & 2 & 2 \\ \text { Copper } & 600 & \text { Blank } & 0 & 0 & 0 & 0 & 0\end{array}$

Time (Min)

$\begin{array}{llllllll}\text { Metal } & \text { Temperature } & \text { Donor } & 1 & 3 & 5 & 7 & 9 \\ \text { Stainless steel } & 600 & 1 & 2 & 1 & 2 & 2 & 1 \\ \text { Stainless steel } & 600 & 2 & 0 & 0 & 1 & 0 & 0 \\ \text { Stainless steel } & 600 & 3 & 3 & 2 & 2 & 2 & 2 \\ \text { Stainless steel } & 600 & \text { blank } & 0 & 0 & 0 & 0 & 0 \\ \text { Stainless steel } & 750 & 1 & 0 & 2 & 2 & 2 & 1 \\ \text { Stainless steel } & 750 & 2 & 0 & 0 & 0 & 0 & 1 \\ \text { Stainless steel } & 750 & 3 & 0 & 2 & 2 & 2 & 2 \\ \text { Stainless steel } & 750 & \text { blank } & 0 & 0 & 0 & 0 & 0 \\ \text { Stainless steel } & 900 & 1 & 2 & 0 & 1 & 1 & 1 \\ \text { Stainless steel } & 900 & 2 & 1 & 0 & 0 & 0 & 0 \\ \text { Stainless steel } & 900 & 3 & 2 & 1 & 2 & 1 & 1 \\ \text { Stainless steel } & 900 & \text { blank } & 0 & 0 & 0 & 0 & 0\end{array}$

Metal Temperature Donor

Brass $200 \quad 1$

Brass $200 \quad 2$

Brass $200 \quad 3$

Brass $200 \quad$ blank

Brass $400 \quad 1$

Brass $400 \quad 2$

Brass $400 \quad 3$

Brass $400 \quad$ blank

Brass $600 \quad 1$

Brass $600 \quad 2$

Brass $600 \quad 3$

Brass $600 \quad$ blank 
Table 6 EDX analysis of oxide fingermark on brass

$\begin{array}{lrrrr}\text { BRASS } & \text { ridge } & \text { hollow } & \text { ridge } & \text { hollow } \\ \mathrm{Cu} & 33.47 \% & 37.84 \% & 32.11 \% & 38.57 \% \\ \mathrm{Zn} & 18.33 \% & 19.08 \% & 16.25 \% & 19.62 \% \\ \mathrm{O} & 38.63 \% & 32.70 \% & 43.46 \% & 32.53 \% \\ \mathrm{Na} & 9.57 \% & 10.38 \% & 8.17 \% & 9.28 \% \\ \mathrm{Cu} /(\mathrm{Cu}+\mathrm{Zn}) & 64.61 \% & 66.48 \% & 66.40 \% & 66.28 \% \\ \mathrm{Zn} /(\mathrm{Cu}+\mathrm{Zn} & 35.39 \% & 33.52 \% & 33.60 \% & 33.72 \% \\ \mathrm{O} /(\mathrm{Cu}+\mathrm{Zn}) & 74.56 \% & 57.44 \% & 89.85 \% & 55.90 \%\end{array}$


Table 7 Fingermark development using ammonium sulphide solution

\begin{tabular}{|c|c|c|c|}
\hline \multicolumn{4}{|c|}{ Copper 0.1 dilution } \\
\hline Sample & Grade & $\begin{array}{l}\text { Time to } \\
\text { appear }\end{array}$ & $\begin{array}{l}\text { Time to } \\
\text { disappear }\end{array}$ \\
\hline 1 & 2 & $1 \min 11 \mathrm{~s}$ & $1 \min 25 \mathrm{~s}$ \\
\hline 2 & 3 & $1 \min 4 \mathrm{~s}$ & $2 \min 28 \mathrm{~s}$ \\
\hline 3 & 1 & $1 \min 35 \mathrm{~s}$ & $2 \min 38 \mathrm{~s}$ \\
\hline
\end{tabular}

\begin{tabular}{|c|c|c|c|}
\hline \multicolumn{4}{|c|}{ Brass 0.1 dilution } \\
\hline Sample & Grade & $\begin{array}{l}\text { Time to } \\
\text { appear }\end{array}$ & $\begin{array}{l}\text { Time to } \\
\text { disappear }\end{array}$ \\
\hline 1 & 1 & $2 \min 30 \mathrm{~s}$ & Did not fade \\
\hline 2 & 1 & $2 \min 45 s$ & Did not fade \\
\hline 3 & 1 & $3 \min 11 \mathrm{~s}$ & Did not fade \\
\hline
\end{tabular}


Table 8 Fingermark development using iodine

Copper

\begin{tabular}{rrrrrr} 
& \multicolumn{5}{c}{ Time $(\min )$} \\
$\begin{array}{rrrrr}\text { Temperature } \\
{ }^{\circ} \mathrm{C}\end{array}$ & Donor & 2 & 4 & 6 & 8 \\
30 & 1 & 0 & 0 & 2 & 2 \\
30 & 3 & 2 & 3 & 3 & 3 \\
40 & 2 & 0 & 0 & 0 & 1 \\
40 & 3 & 1 & 2 & 2 & 2 \\
50 & 1 & 1 & 2 & 2 & 2 \\
50 & 2 & 0 & 0 & 0 & 0
\end{tabular}

\begin{tabular}{|c|c|c|c|c|c|}
\hline \multirow[b]{2}{*}{$\begin{array}{r}\text { Temperature } \\
{ }^{\circ} \mathrm{C}\end{array}$} & Brass & \multicolumn{4}{|c|}{ Time (min) } \\
\hline & Donor & 2 & 4 & 6 & 8 \\
\hline 30 & 1 & 1 & 2 & 2 & 2 \\
\hline 30 & 2 & 0 & 1 & 1 & 1 \\
\hline 40 & 1 & 2 & 3 & 3 & 3 \\
\hline 40 & 3 & 2 & 3 & 4 & 4 \\
\hline 50 & 2 & 1 & 1 & 1 & 2 \\
\hline 50 & 3 & 2 & 3 & 3 & 4 \\
\hline
\end{tabular}


Table 9 Fingermark enhancement using Fry's reagent

\begin{tabular}{lllllll} 
& \multicolumn{7}{c}{ Before } & & After \\
& Grade 0 & Grade 1 & Grade 3 & Grade 0 & Grade 1 & Grade 3 \\
& & or 2 & or 4 & & or 2 & or 4 \\
Brass & 0 & 17 & 15 & 0 & 8 & 24 \\
Copper & 3 & 8 & 7 & 5 & 8 & 5 \\
Tinned & 9 & 7 & 6 & 2 & 5 & 15 \\
steel & & & & & & \\
Stainless & 5 & 12 & 5 & 8 & 7 & 7 \\
steel & & & & & & \\
Total & 17 & 44 & 33 & 15 & 28 & 51
\end{tabular}


Figure 1 Brass 5 min at $380{ }^{\circ} \mathrm{C}$ by confocal microscope a) showing detail of pore contact and b) $2 \times 2$ stitched image showing larger area of mark

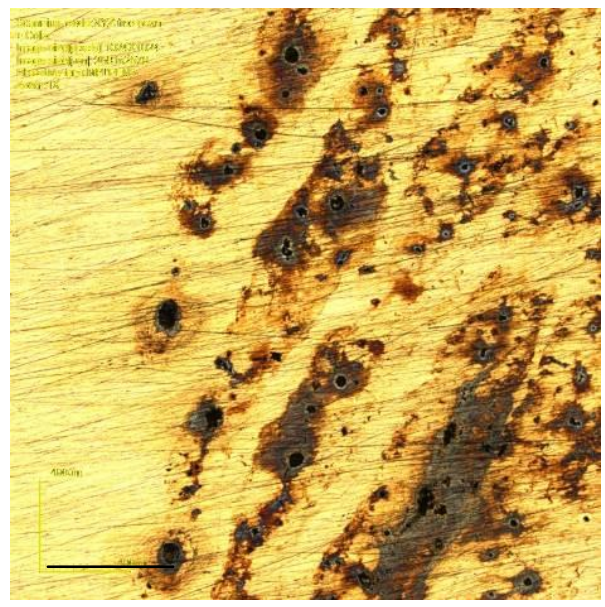

$500 \mu \mathrm{m}$ scale

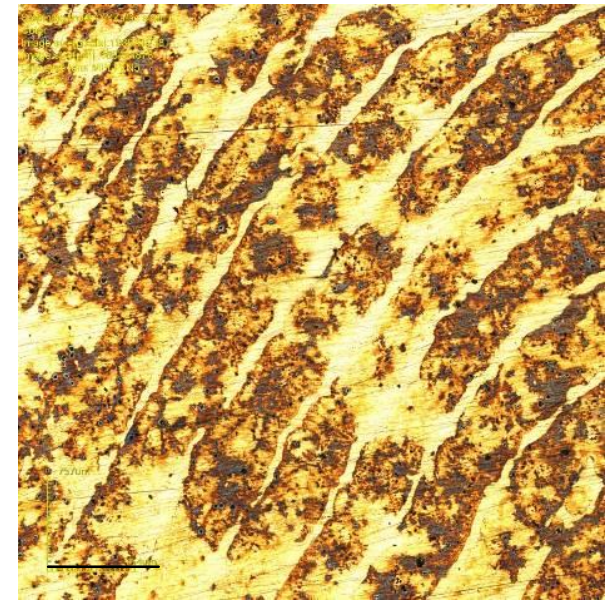

$750 \mu \mathrm{m}$ scale 
Figure 2 Confocal microscope image of fingermark on brass heated at $380{ }^{\circ} \mathrm{C}$ for 5 min showing 2D image, 3D image with extended z-axis, and depth profile

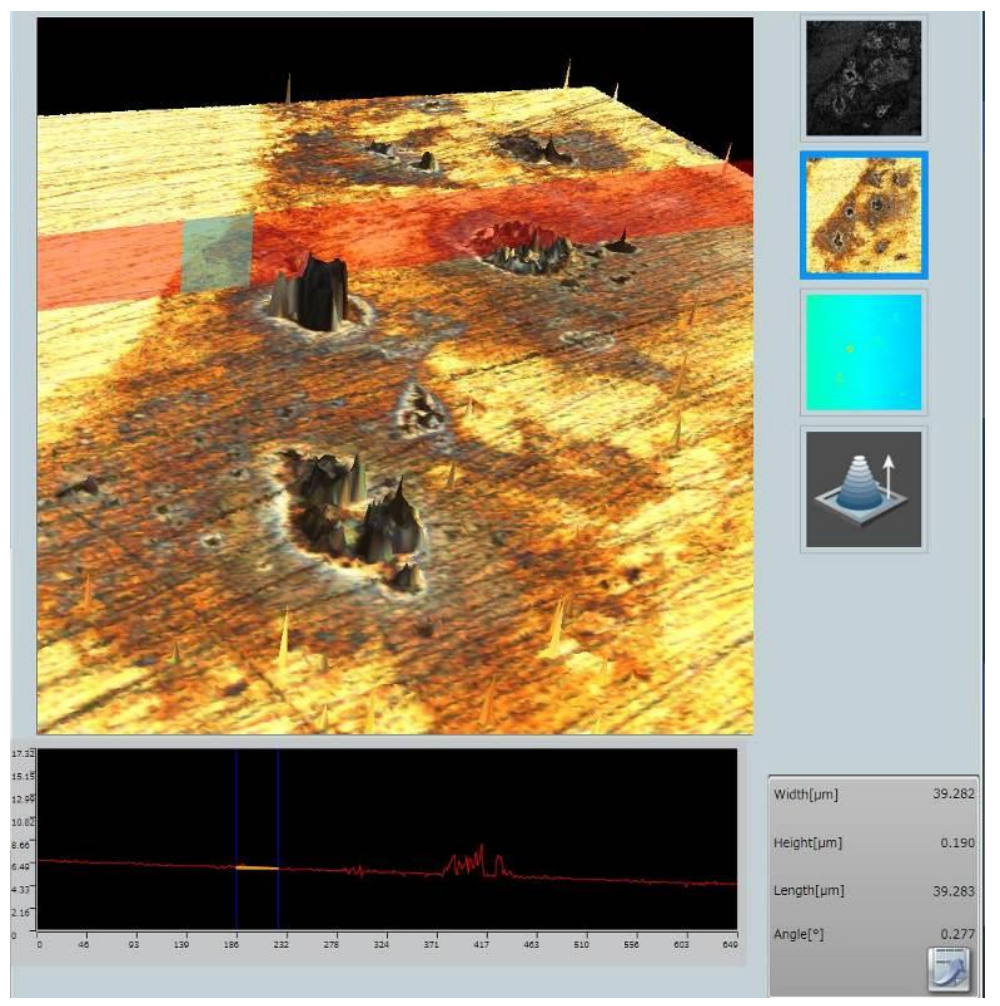


Figure 3 Confocal microscope image of salt solution on brass heated at $400{ }^{\circ} \mathrm{C}$ for 5 min showing 2D image, 3D image with extended z-axis, and depth profile

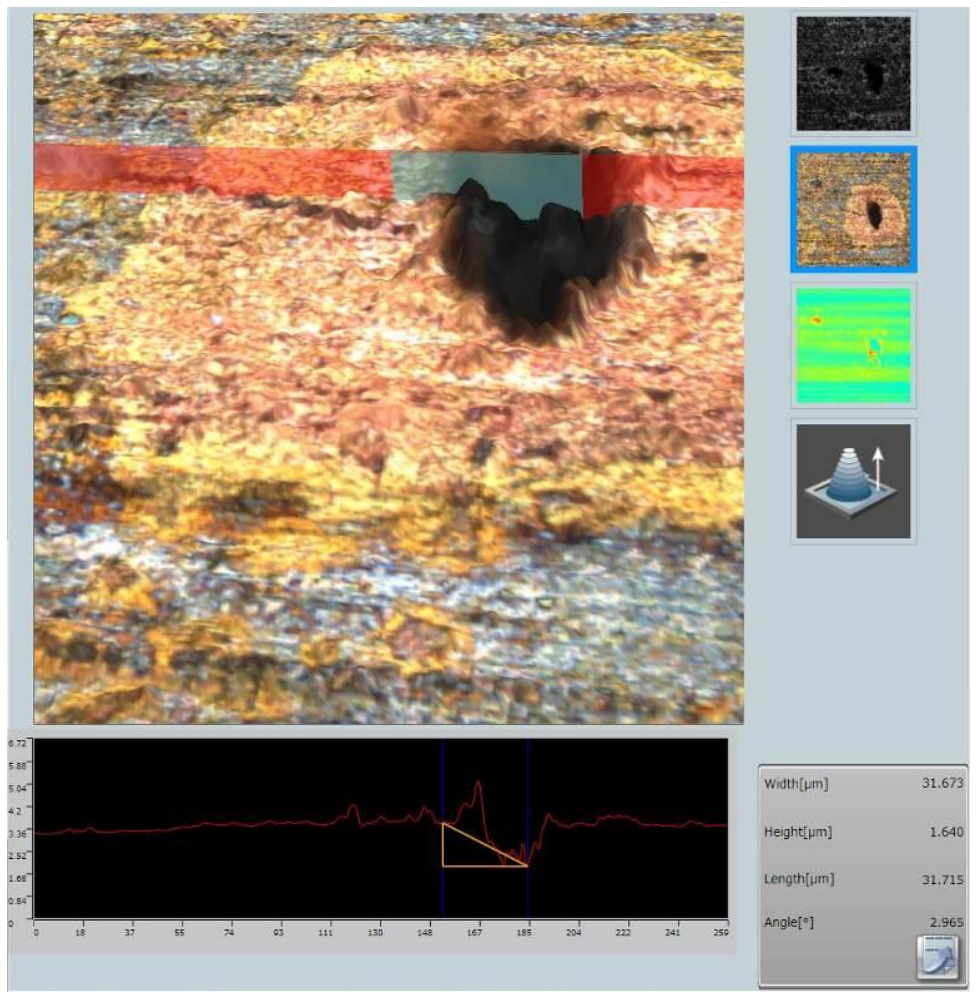


Figure 4 SEM EDX image of fingermark on brass heated for 5 min at $600{ }^{\circ} \mathrm{C}$ : SEM image, oxygen, sodium, copper, zinc concentrations.

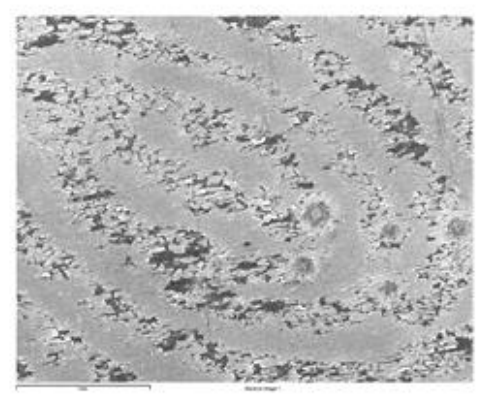

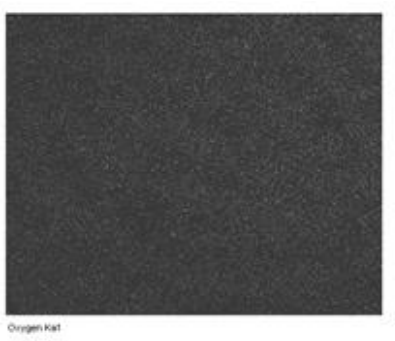

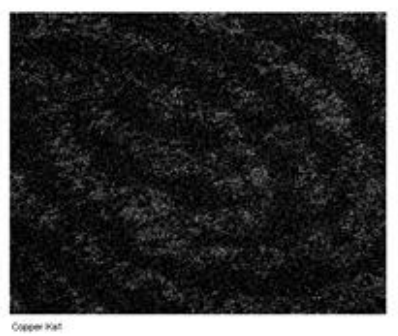

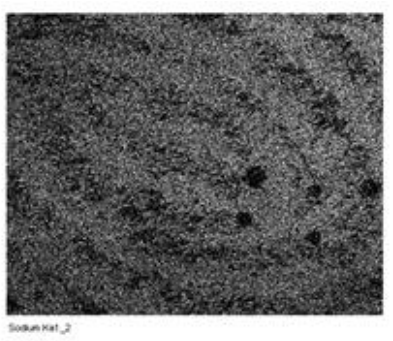

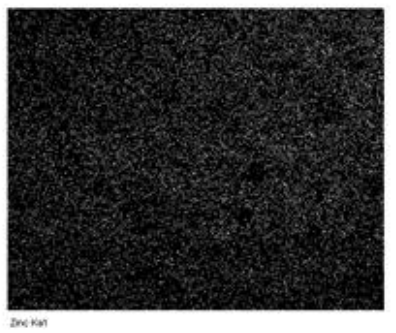


Figure 5 Mild steel with fingermark and heated for $5 \mathrm{~min}$ at $600{ }^{\circ} \mathrm{C}$, confocal microscope: a) 2D image showing dendritic growth, b) stitched 3D image with extended z-axis showing ridges, and c) 3D image of pore with enhanced z-axis
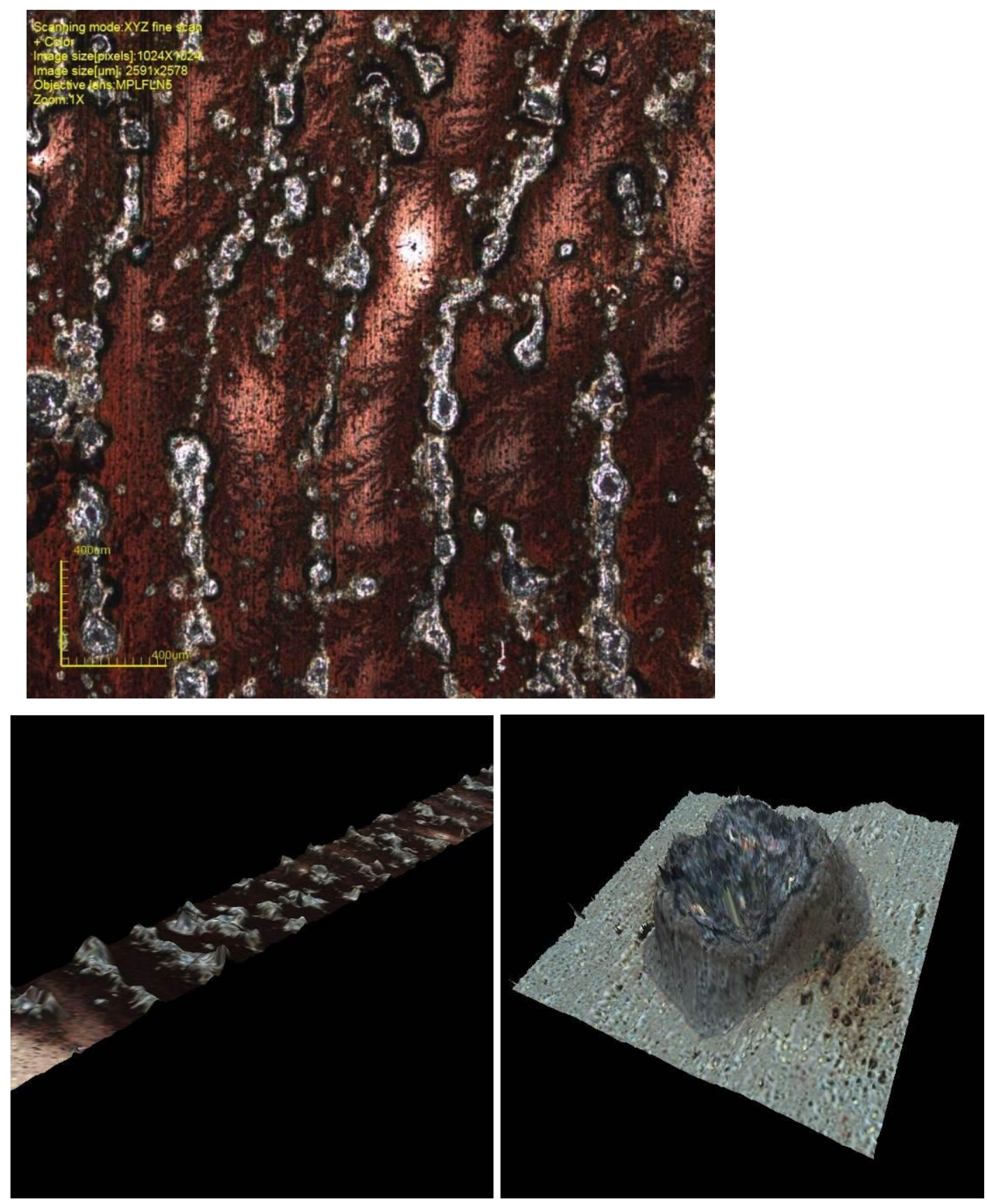
Figure 6 Copper oxide fragmentation showing black $\mathrm{CuO}$, red $\mathrm{Cu}_{2} \mathrm{O}$ and metallic copper.

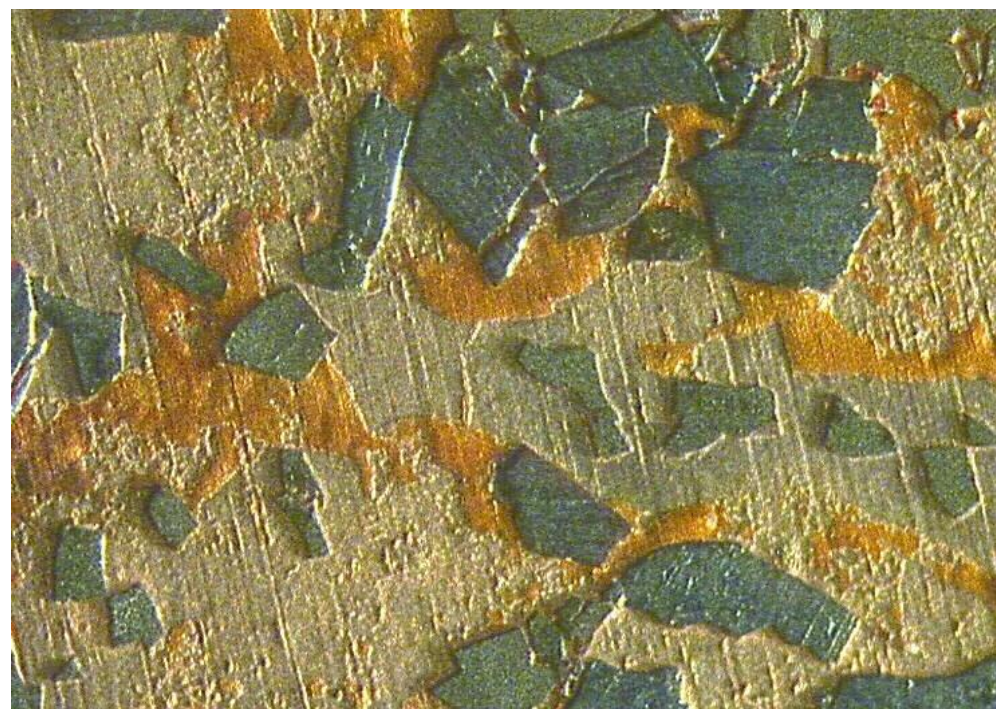


Figure 7 Confocal microscope images of copper heated for 5 min at $600{ }^{\circ} \mathrm{C}$ : a) showing oxide fracture coincident with fingermark, b) 3D image with extended zaxis and oxide thickness measurement, and c) 3D image showing raised section in oxide of possible pore
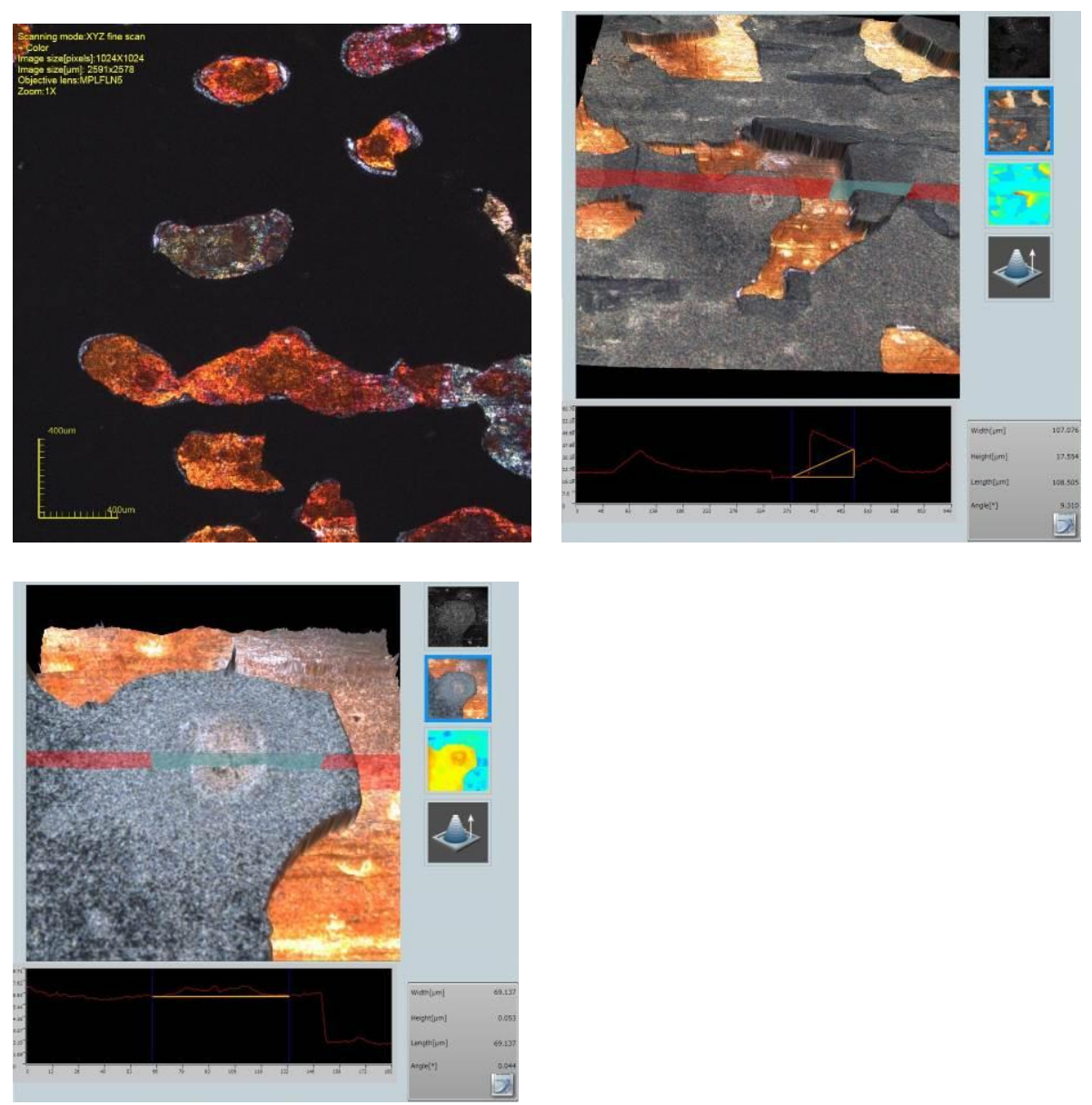
Figure 8 SEM image of copper with fingermark showing oxide layer and appearance of fingermark through the oxide

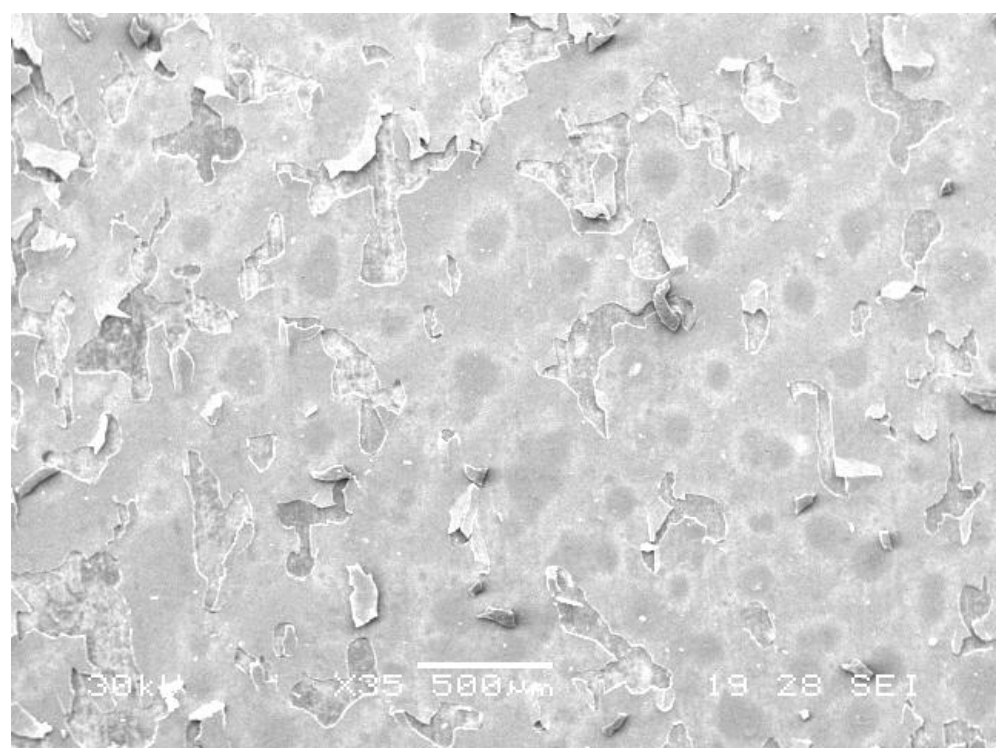


Figure 9: Copper heated at $800{ }^{\circ} \mathrm{C}$ for $5 \mathrm{~min}$ a) before lift showing slight evidence of fingermark, b) after oxide lift showing fingermark on underlying metal, c) copper heated to 600 .
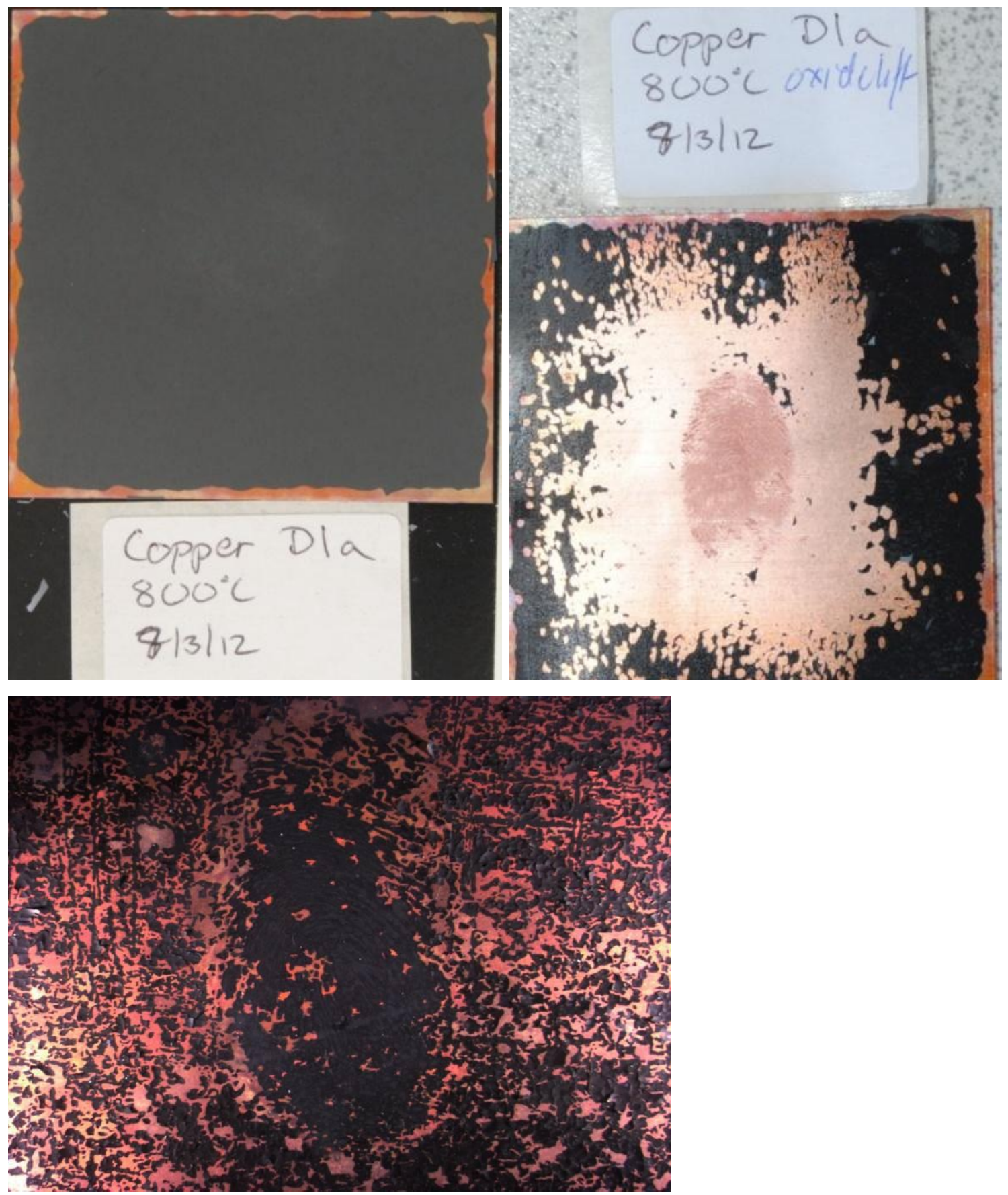
Figure 10: Recovered fingermarks by tape lift from oxidised metal: a) copper heated at and b) $500{ }^{\circ} \mathrm{C}$ brass heated at $800^{\circ} \mathrm{C}$

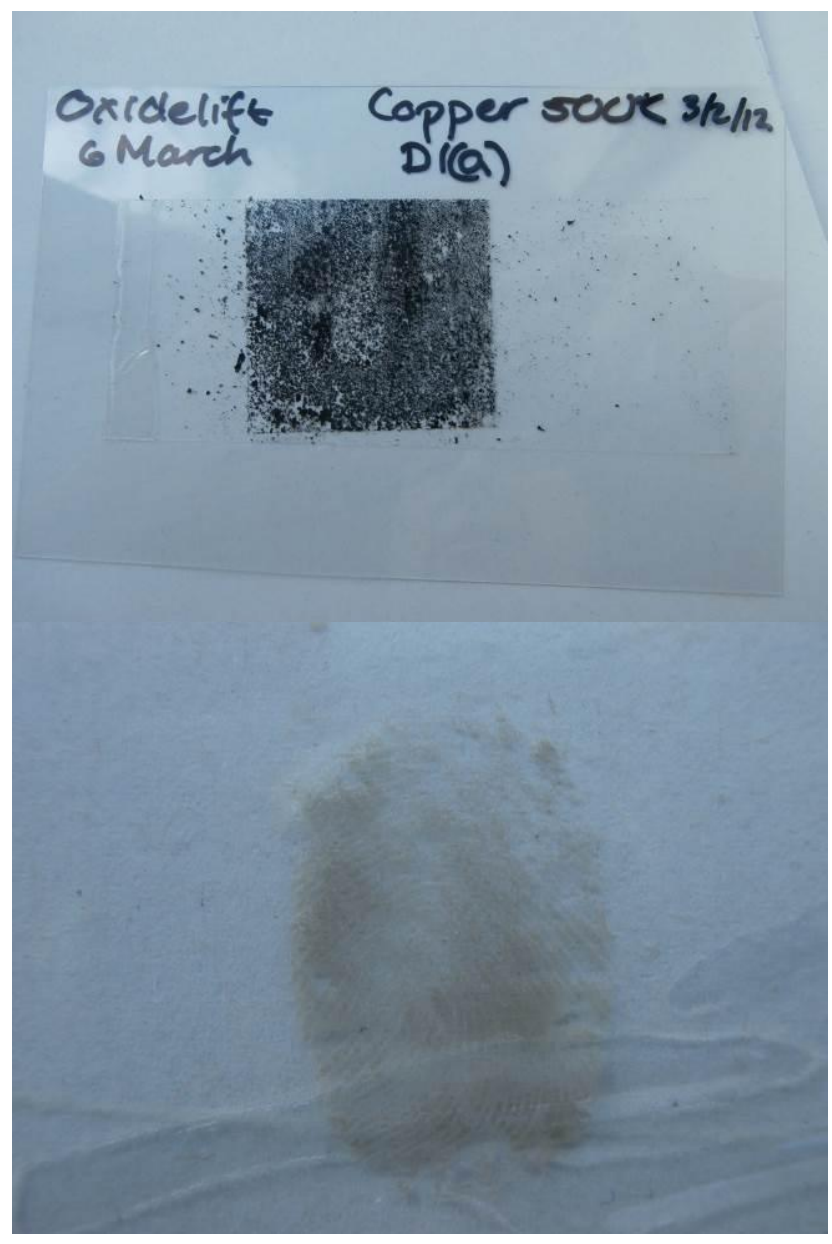


Figure 11 Fingermark on copper treated with 10 -fold dilution of ammonium sulphide solution

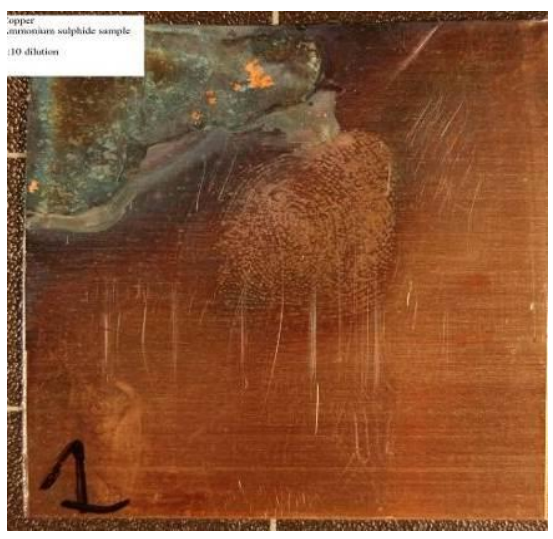


Figure 12 SEM image of copper treated with iodine vapour a) x35 and b) x 900 magnification)
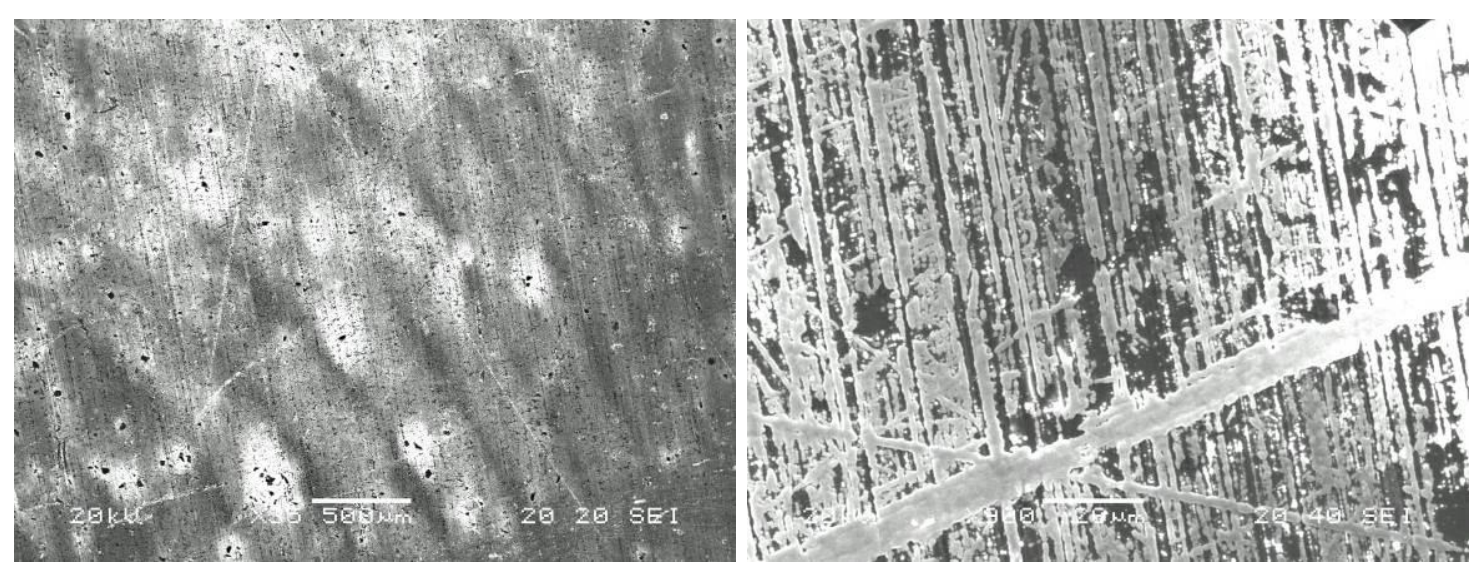
Figure 13 Fingermarks on copper: a) hydrogen peroxide solution, and b) carbamide peroxide solution with eccrine mark on the left and sebaceous-loaded mark on the right
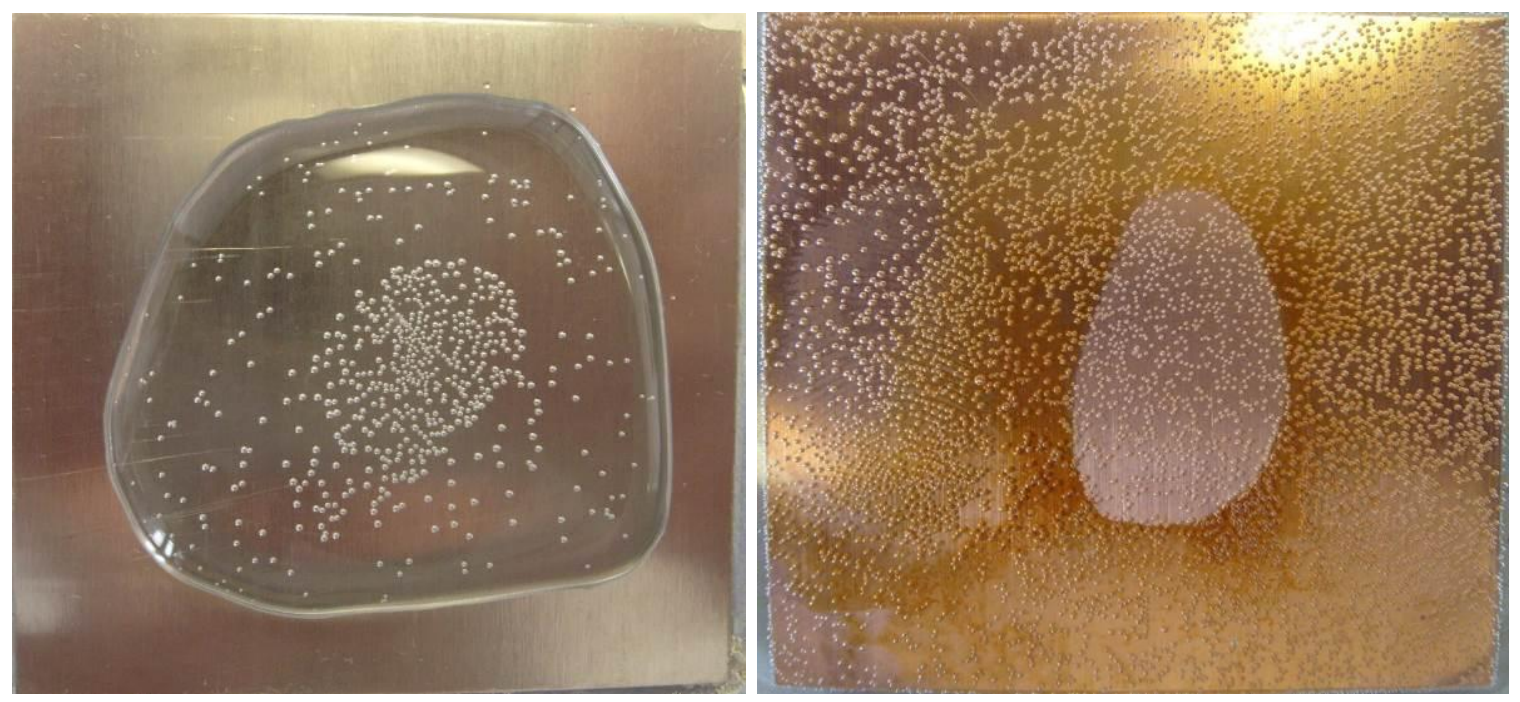
Figure 14 Anodised metal samples: a) fingermark on tinned steel, and b) aluminium treated with anodising dye. Fingermarks were half submerged during processing to differentiate treated and untreated marks.

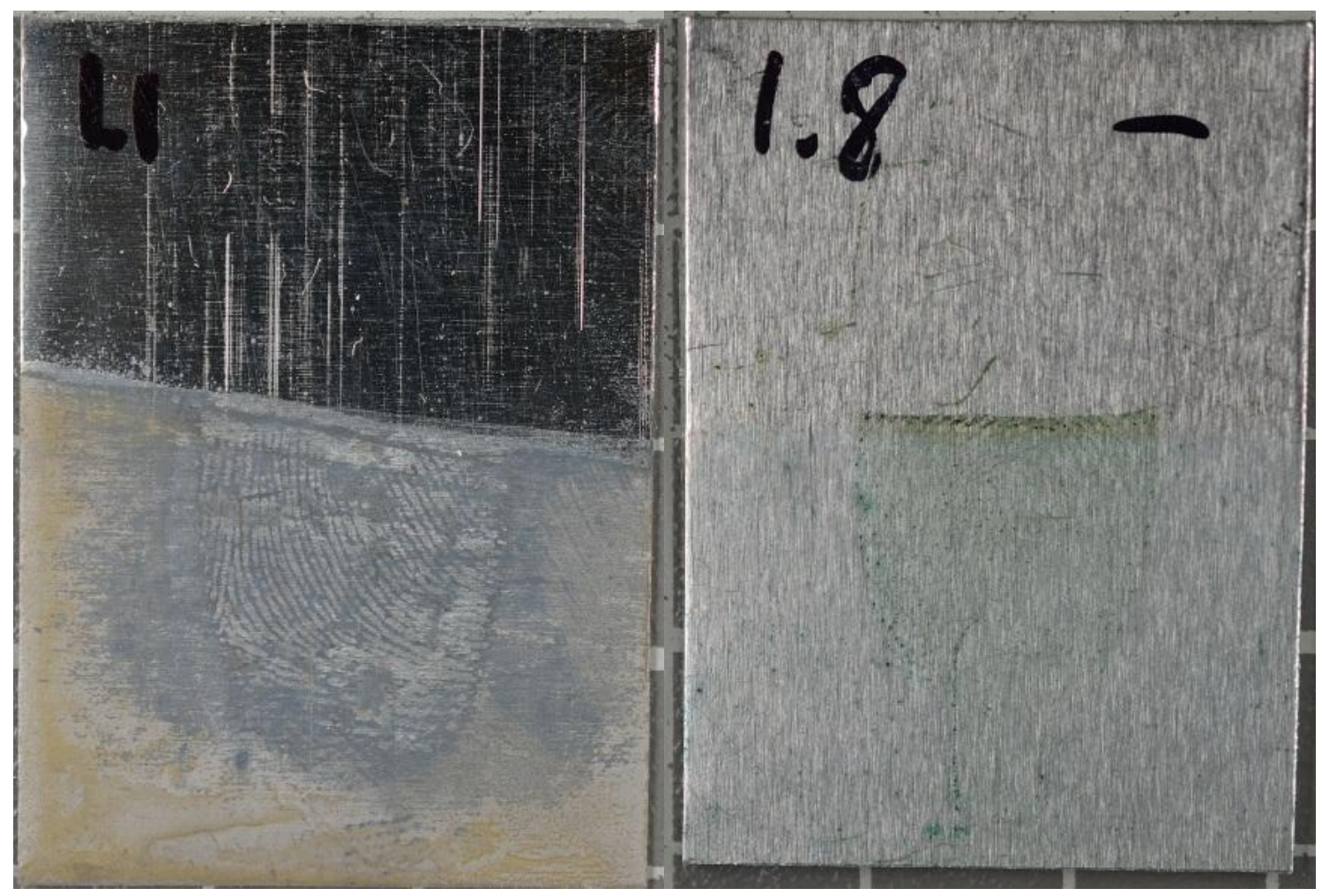


Figure 15a Fingermarks on mild steel submerged in salt water (3 donors) and figure $15 \mathrm{~b}$ anodised tinned steel treated with water
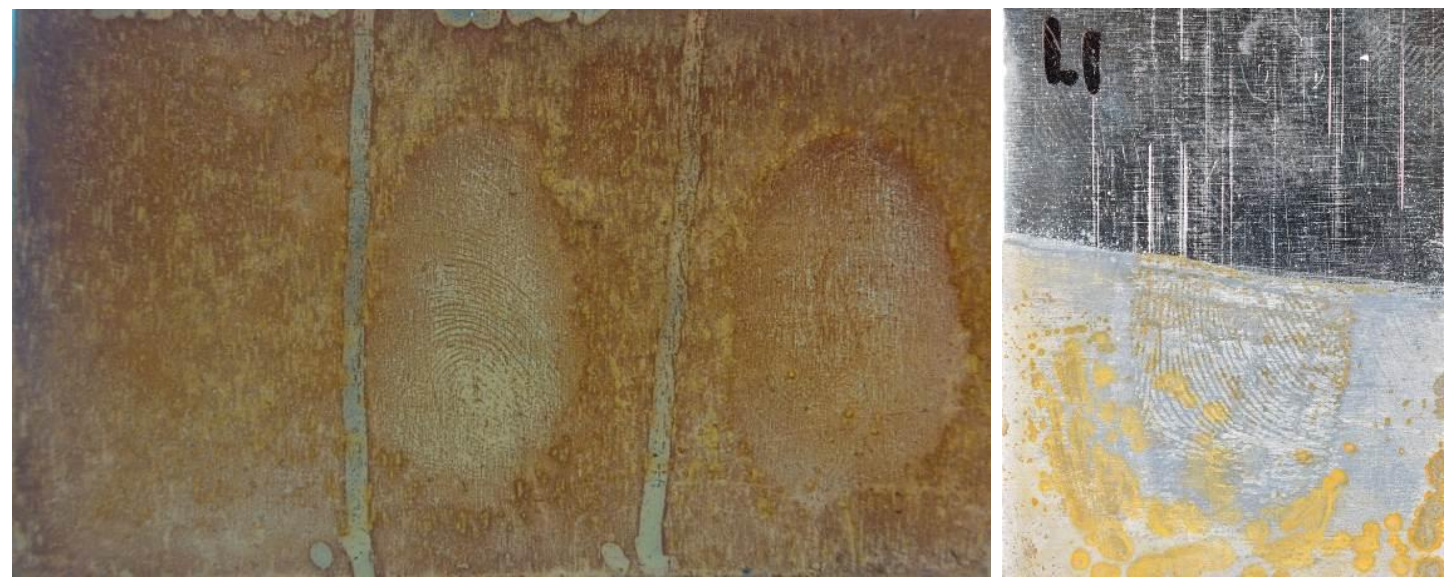


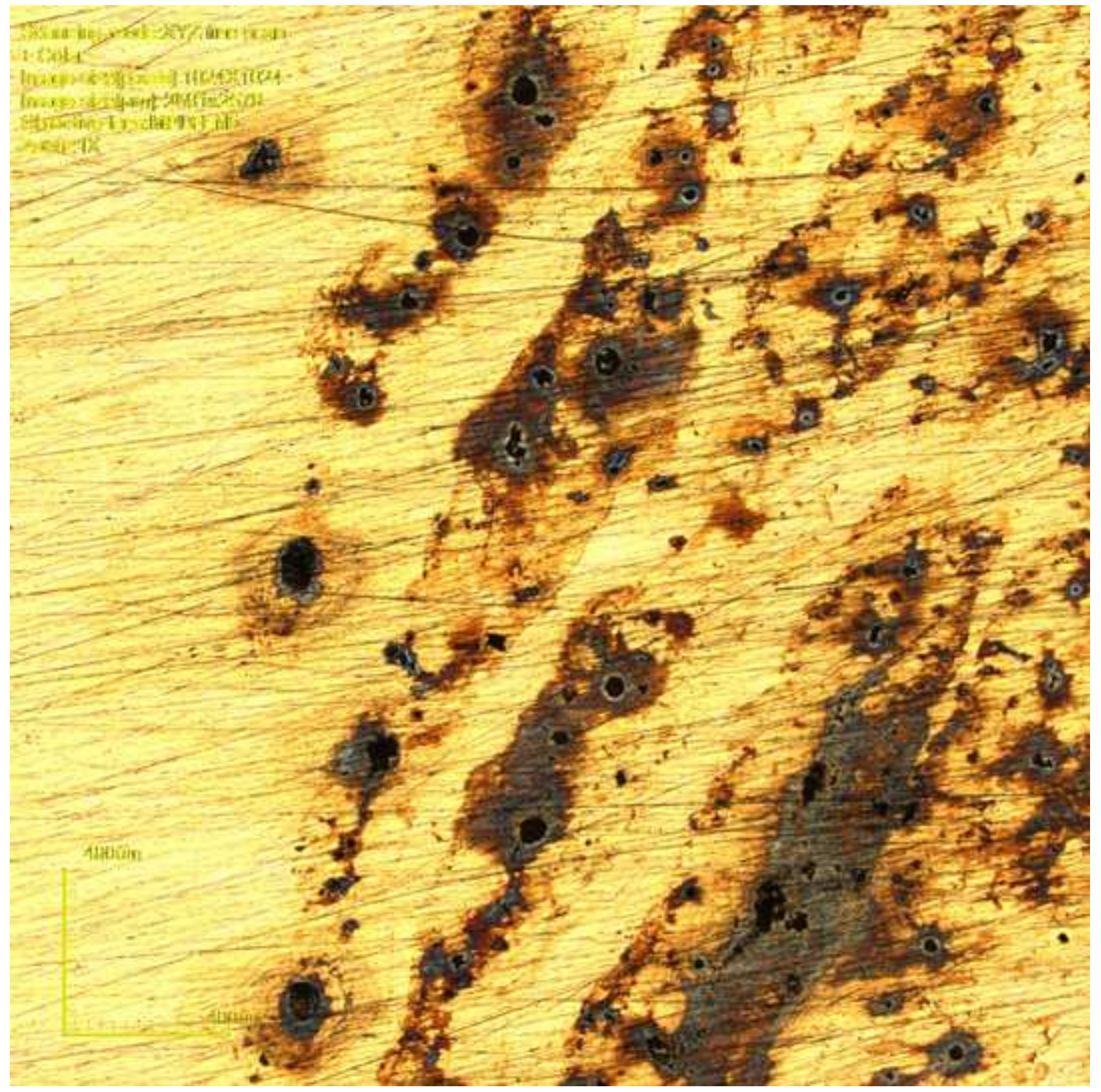




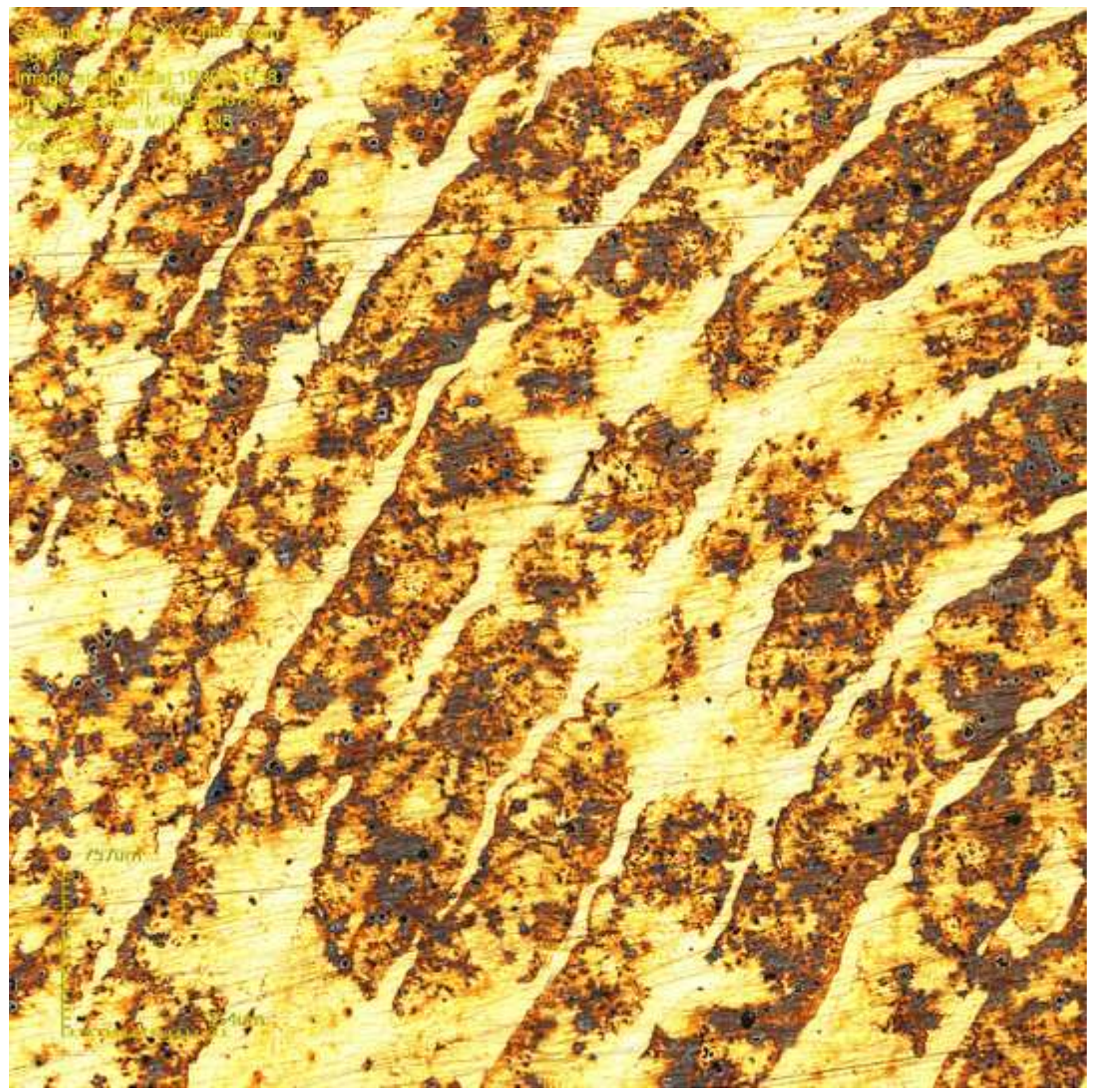


Click here to download high resolution image

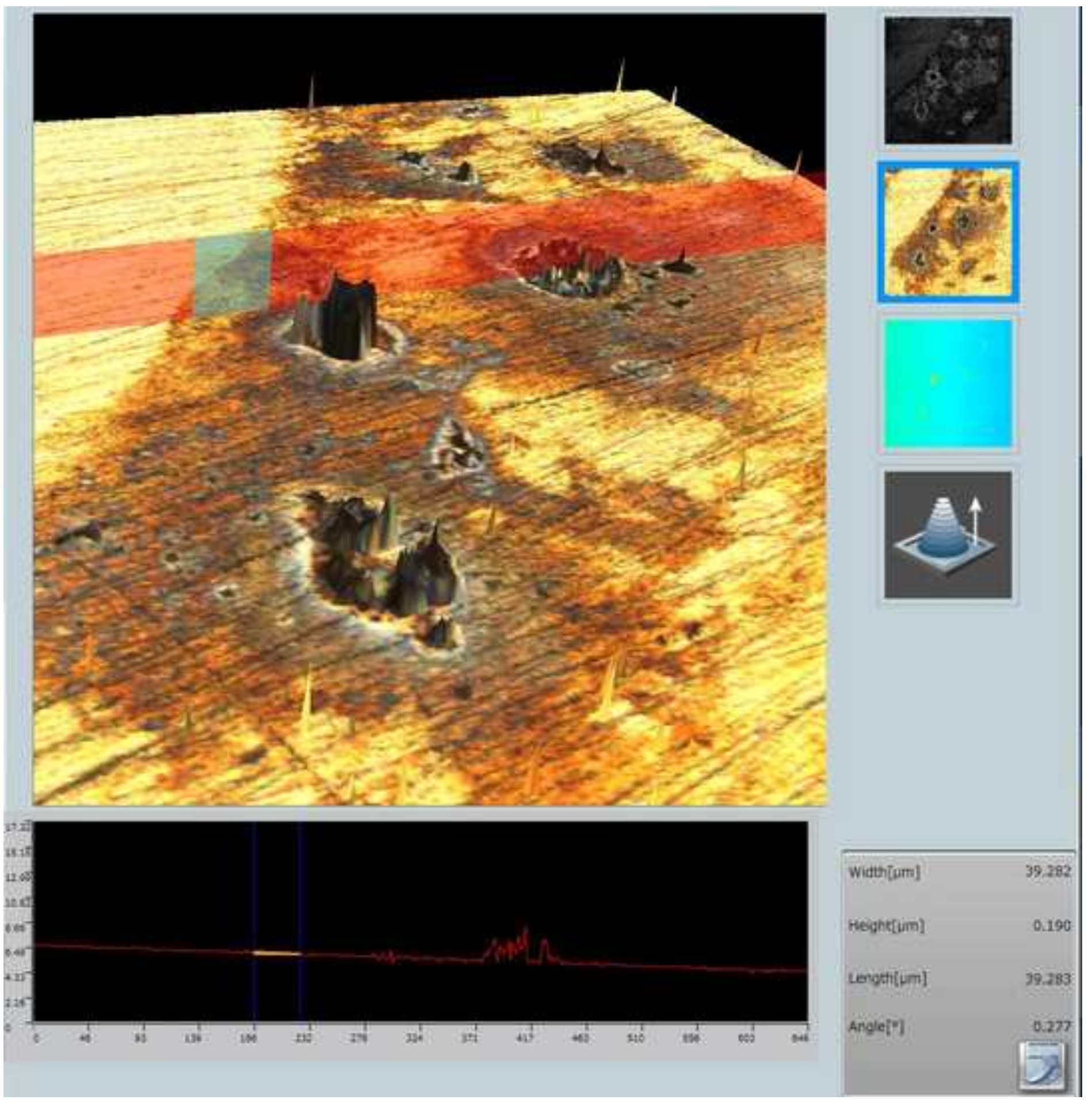




\section{Click here to download high resolution image}
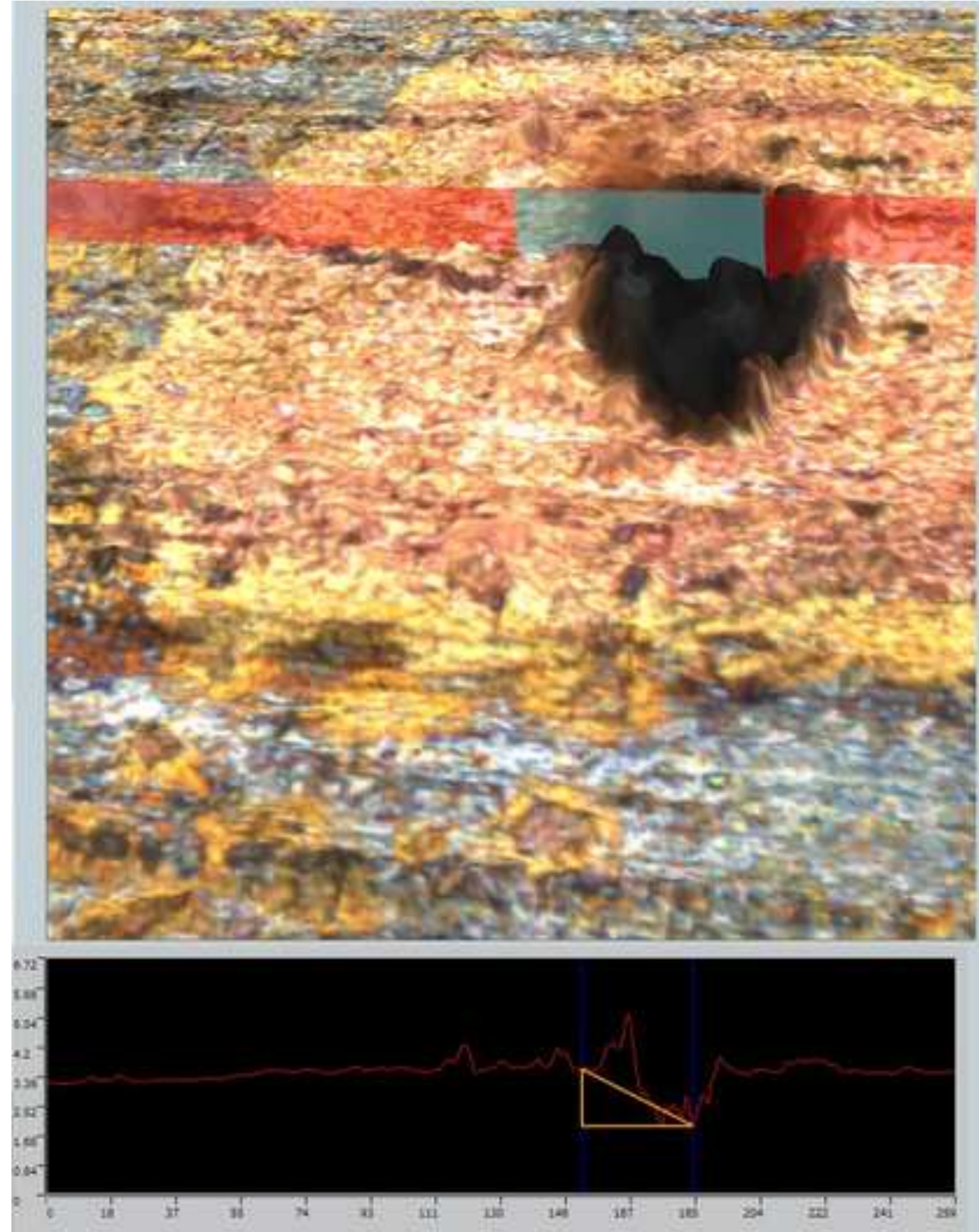

\begin{tabular}{lr}
\hline Width[um] & 31.673 \\
Heignitum] & 1.640 \\
Lengthlim) & 31.715 \\
Angiet'] & 2.955 \\
& -27 \\
\hline
\end{tabular}
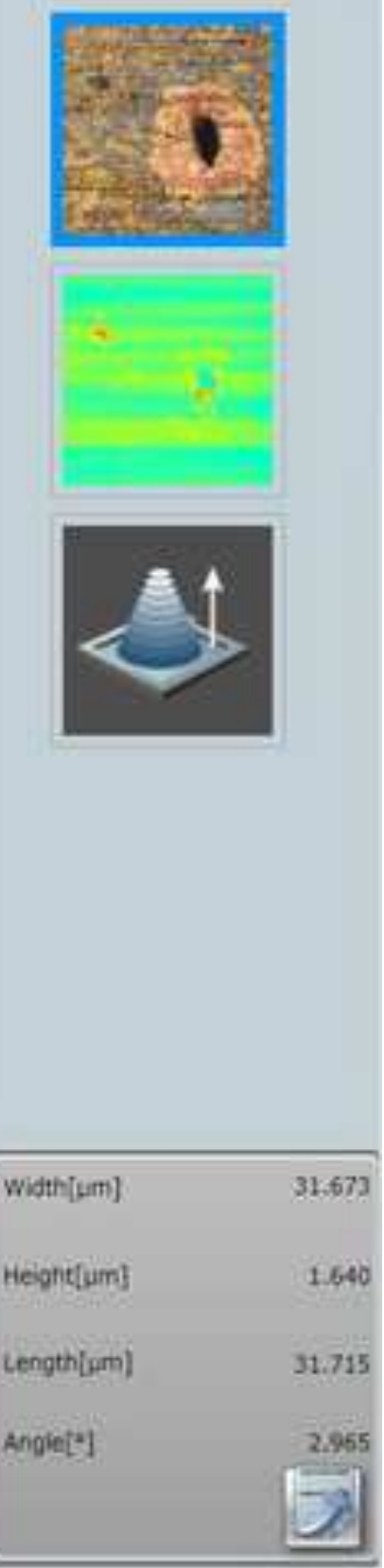


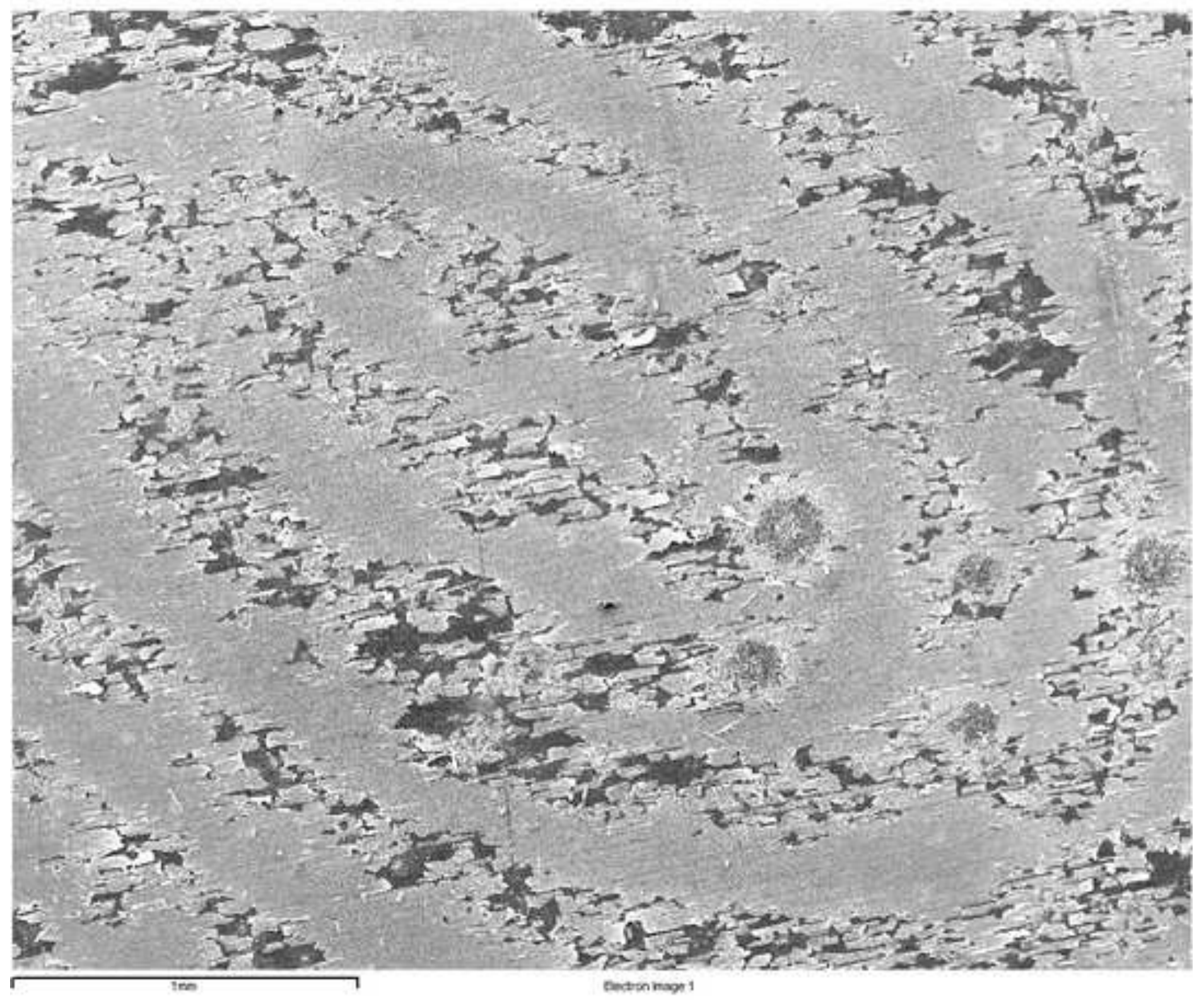




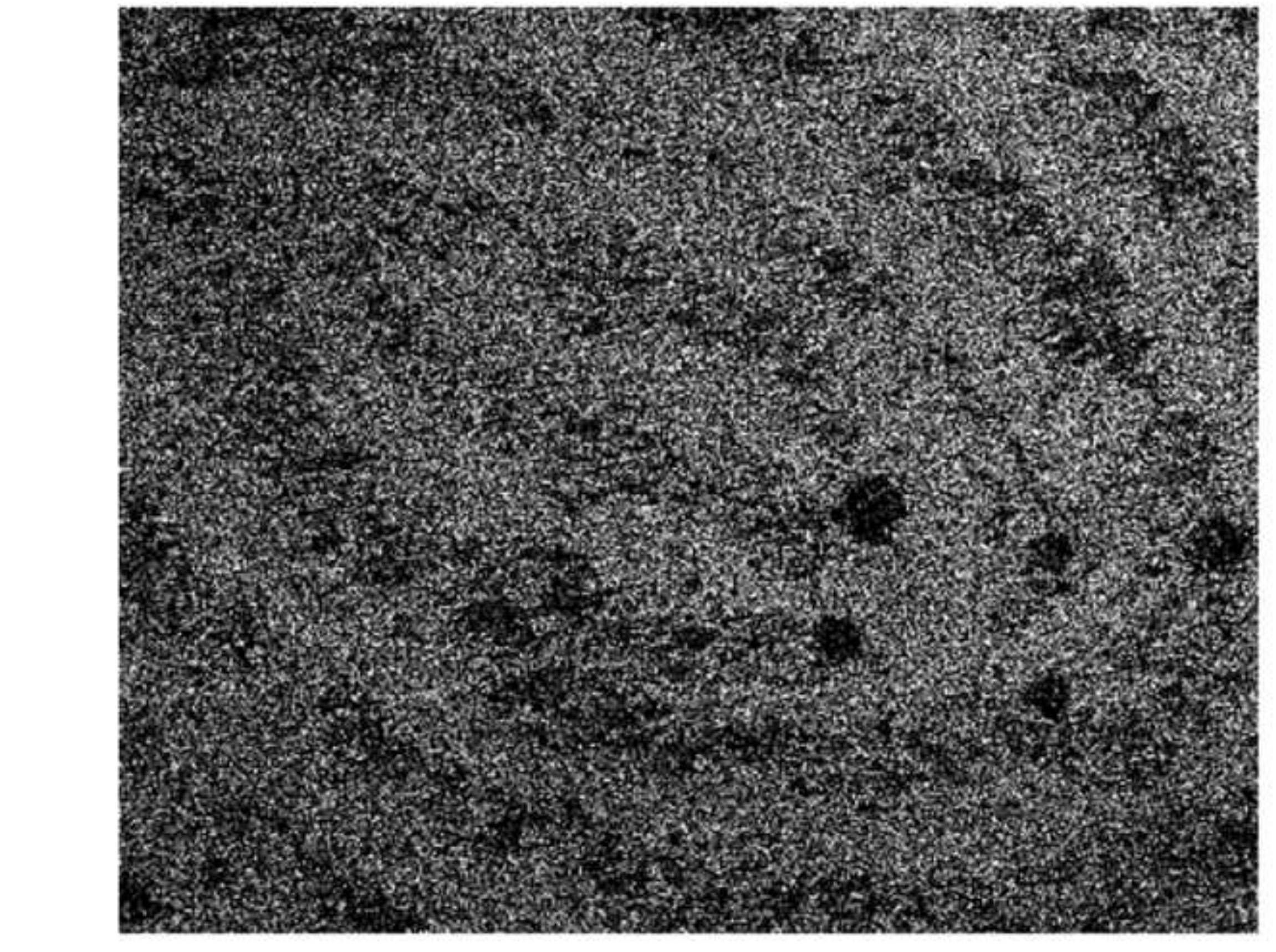

Sodium Ka1_2

\section{(1)}
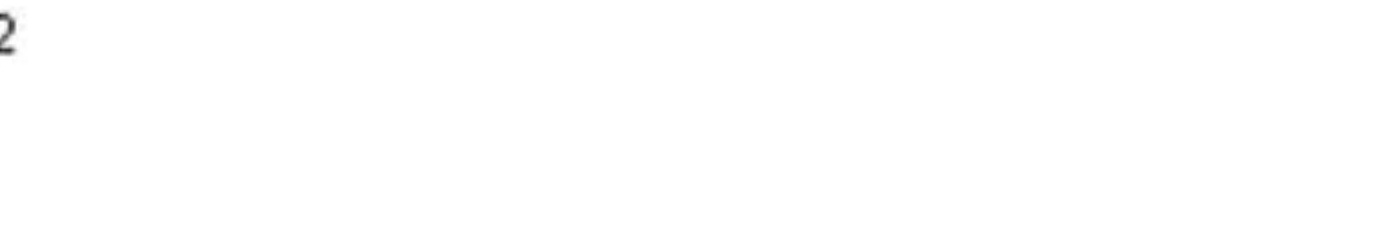


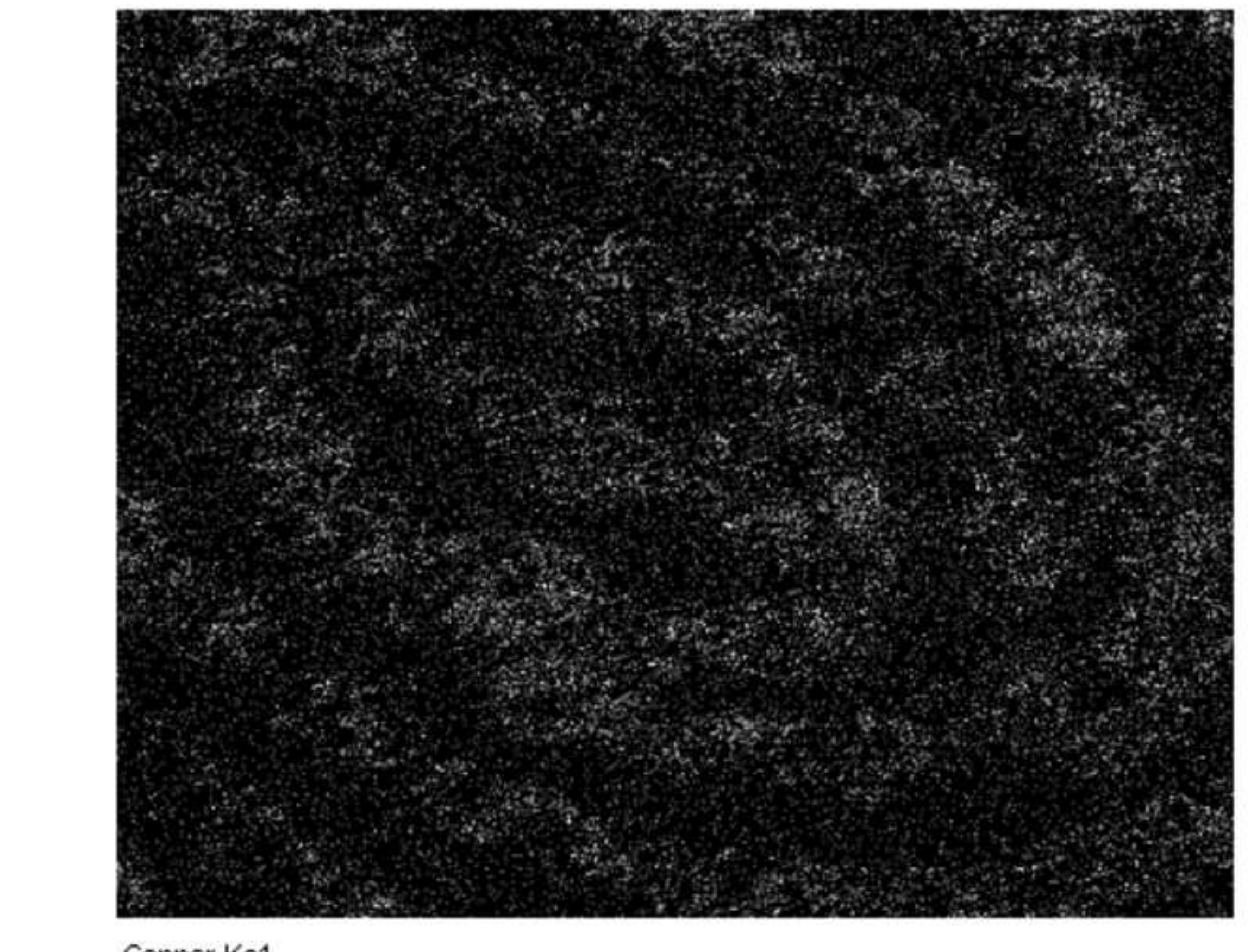

\section{Copper Ka1}

\section{Click here to download high resolution image}

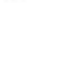

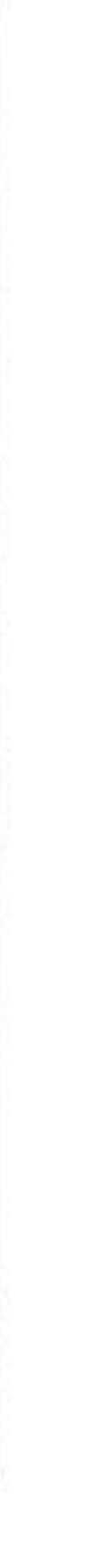




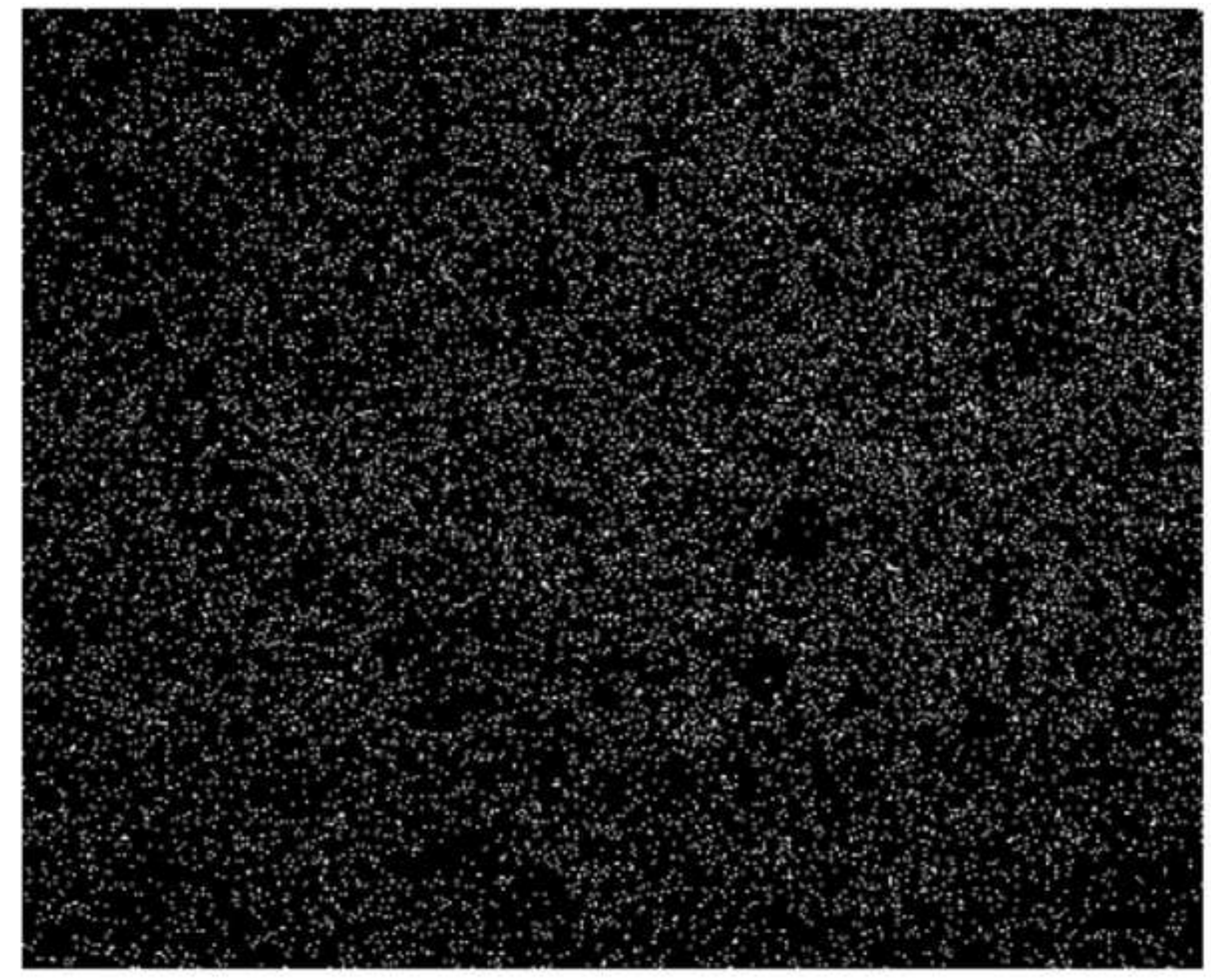

\section{Zinc Ka1}




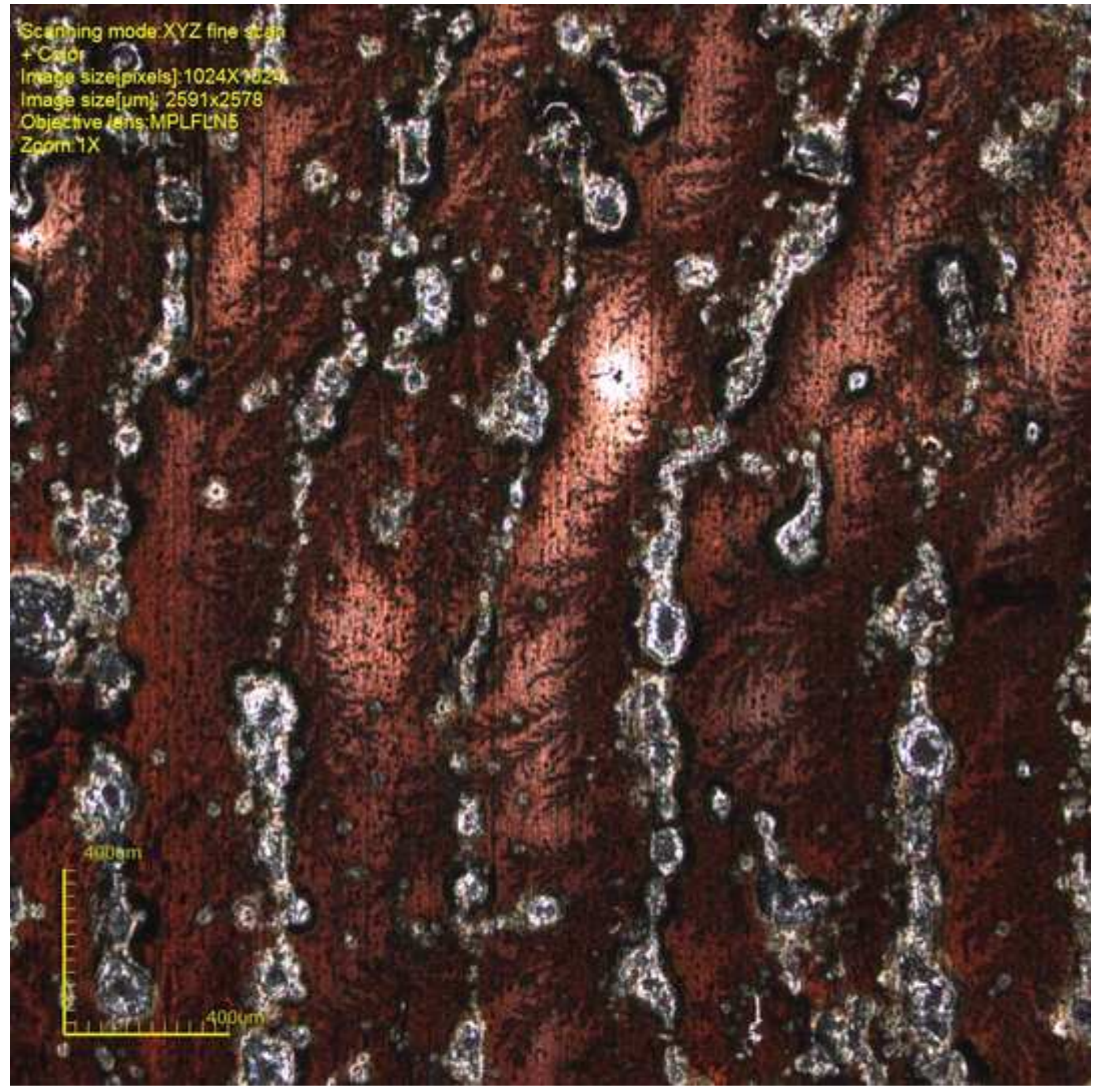


Figure 5b

Click here to download high resolution image

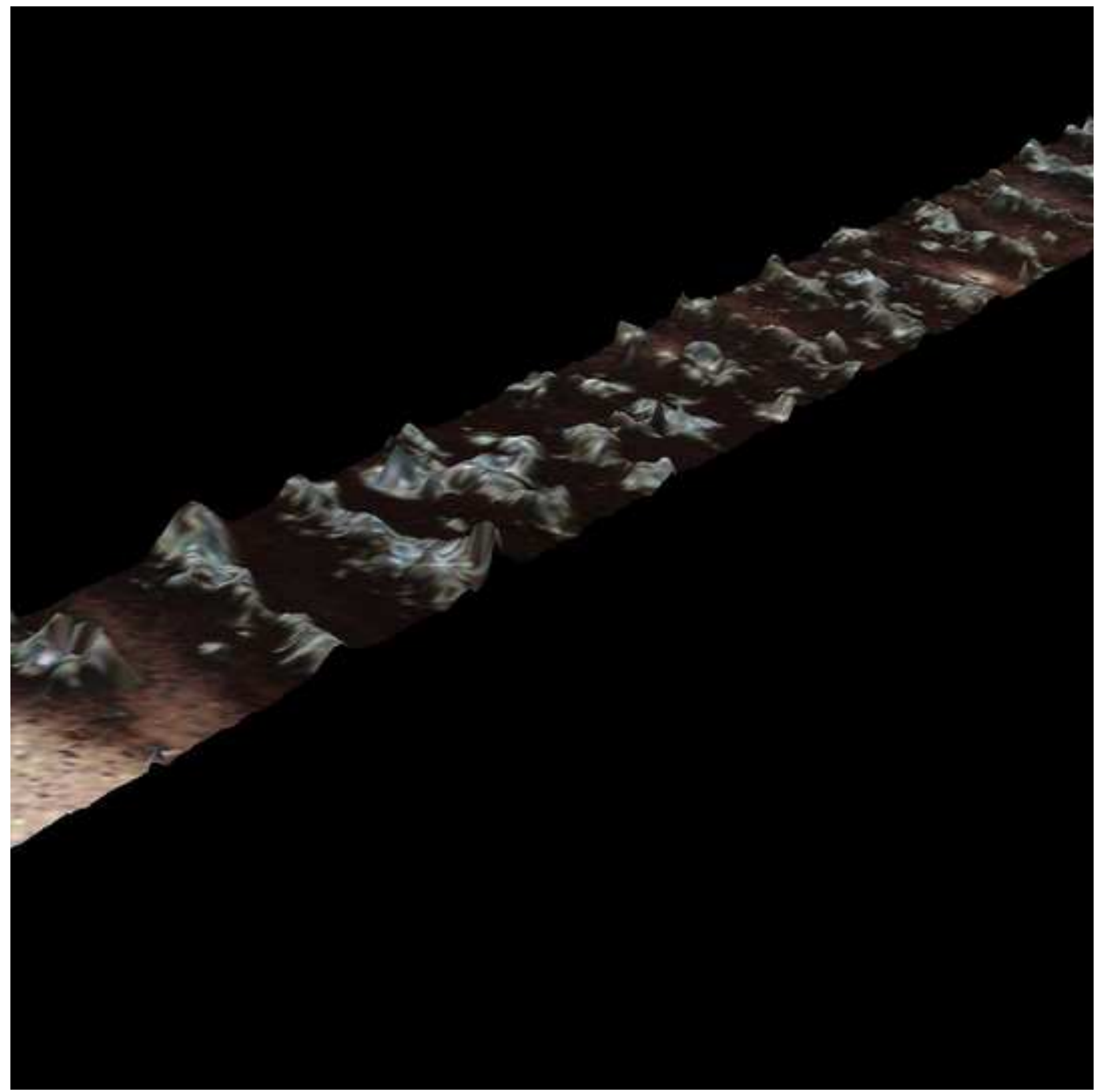


Figure $5 c$
Click here to download high resolution image

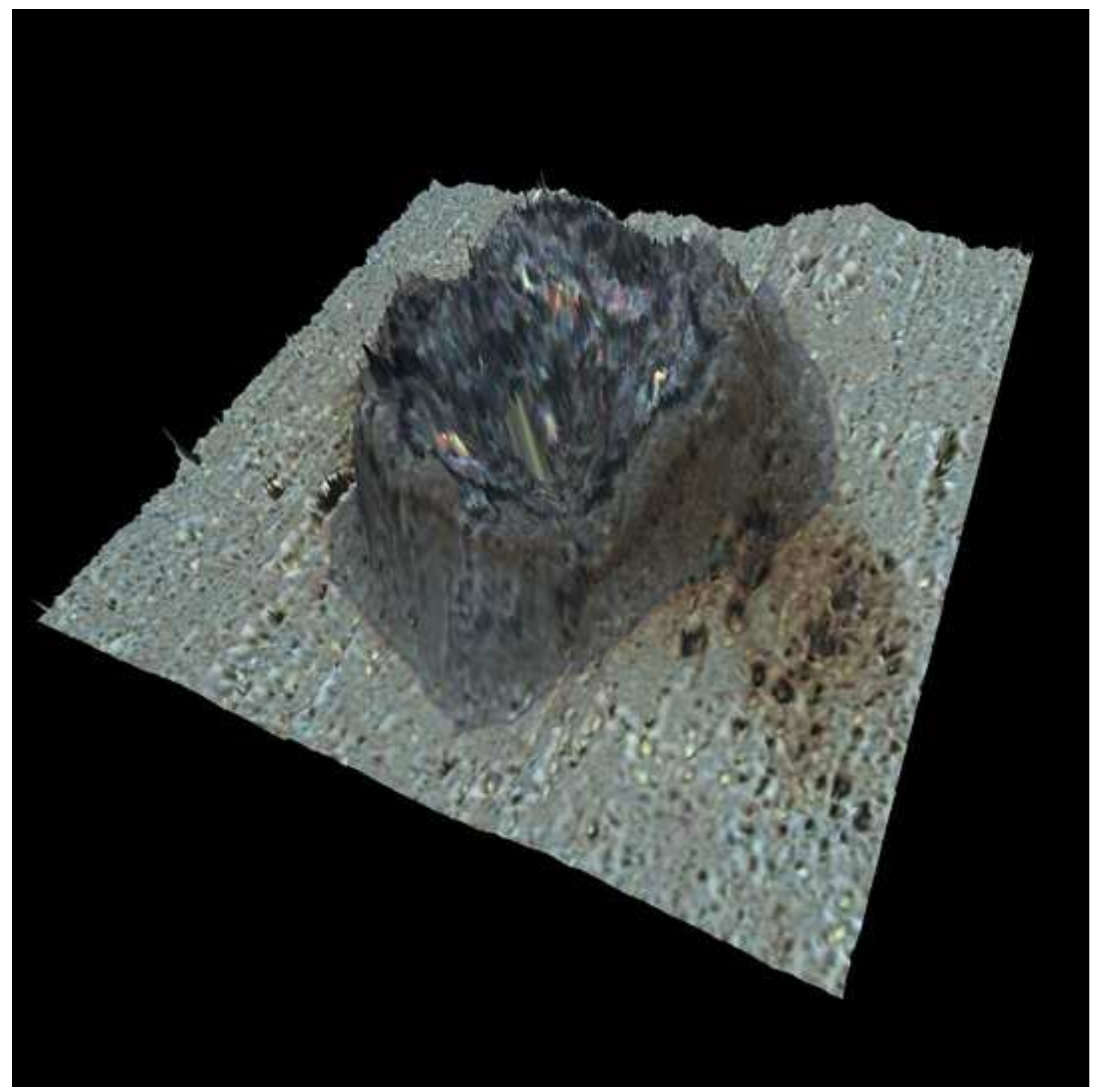


Figure $7 a$
Click here to download high resolution image

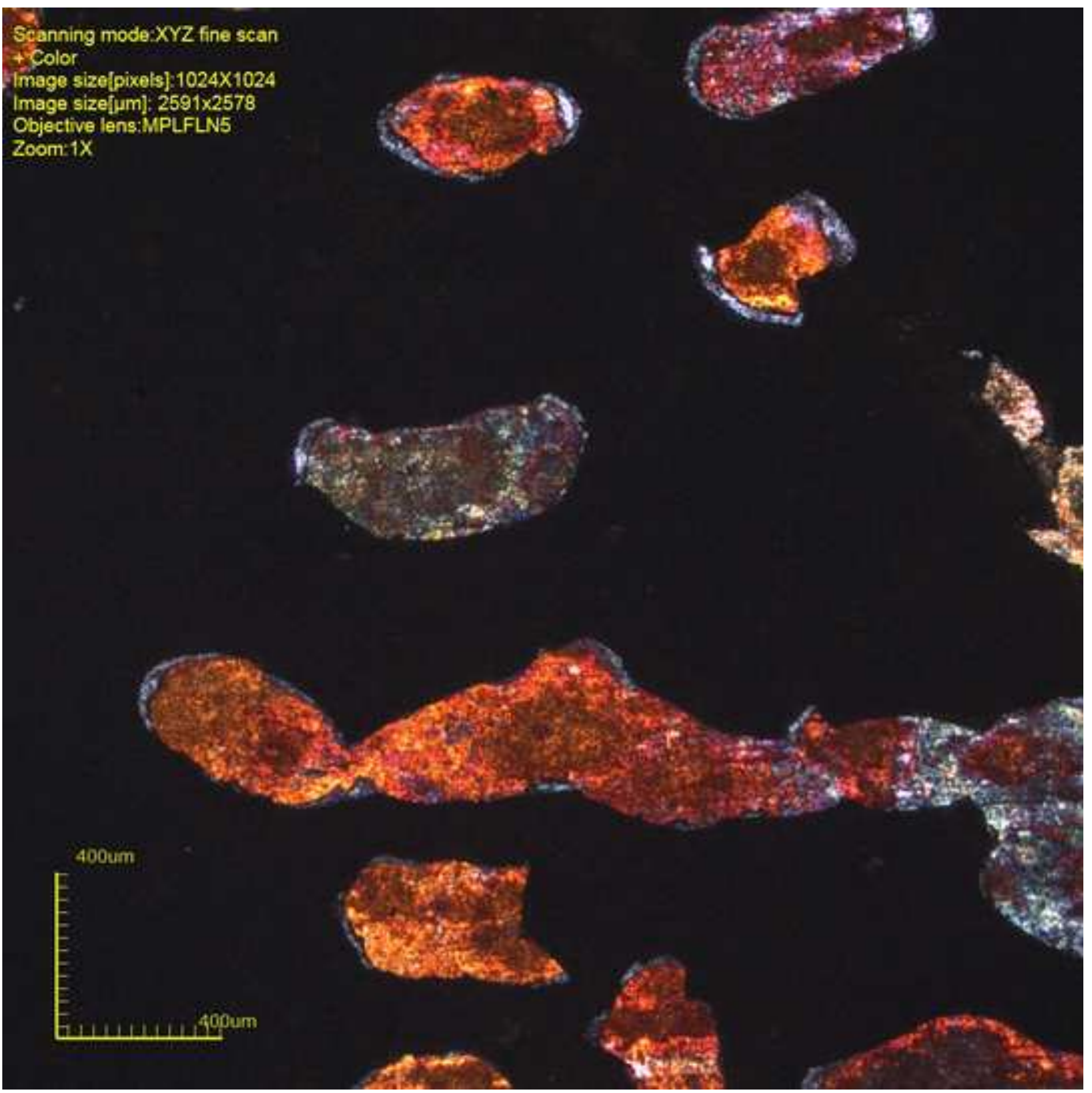


Click here to download high resolution image
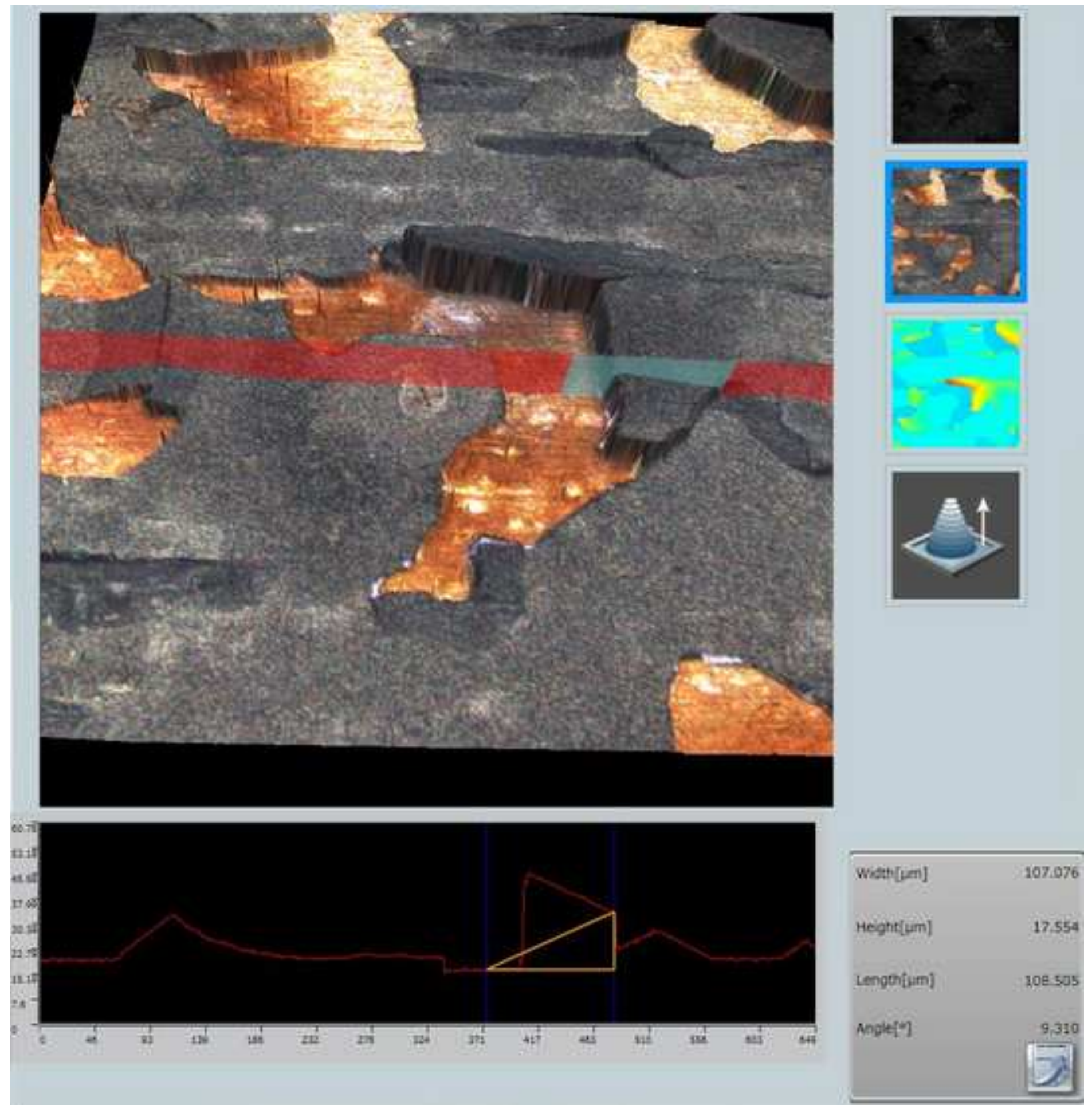
Click here to download high resolution image
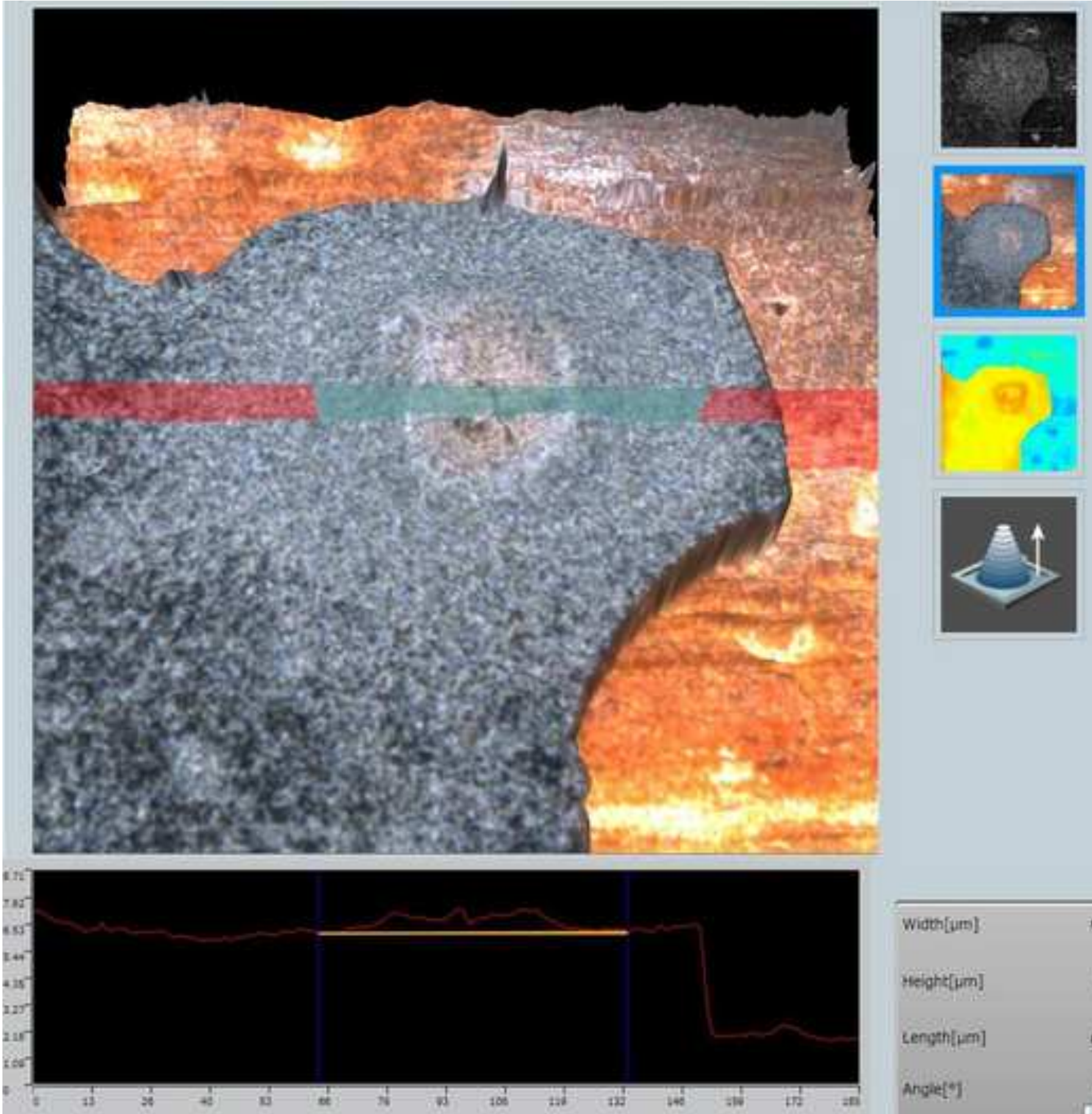
Click here to download high resolution image

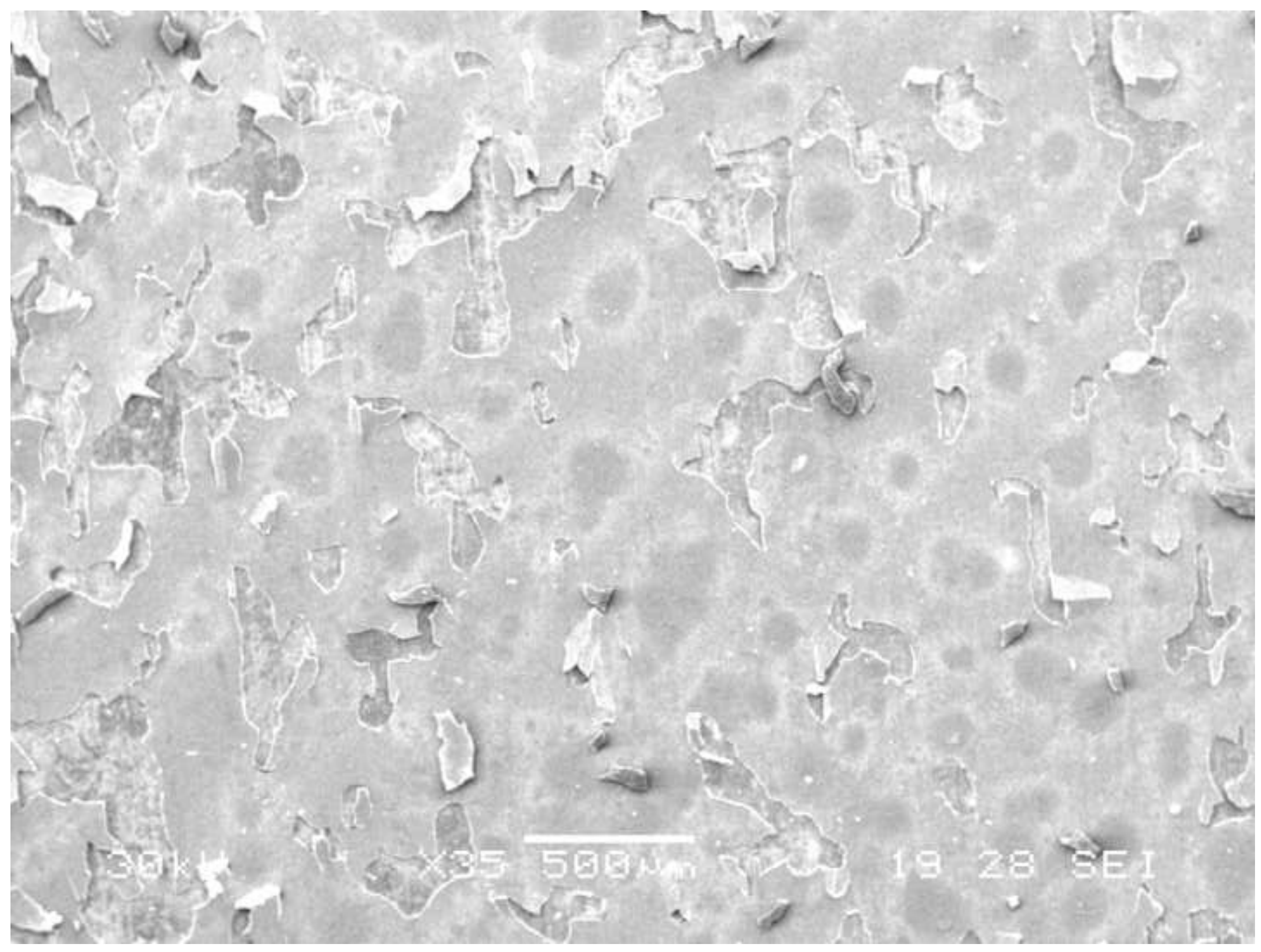




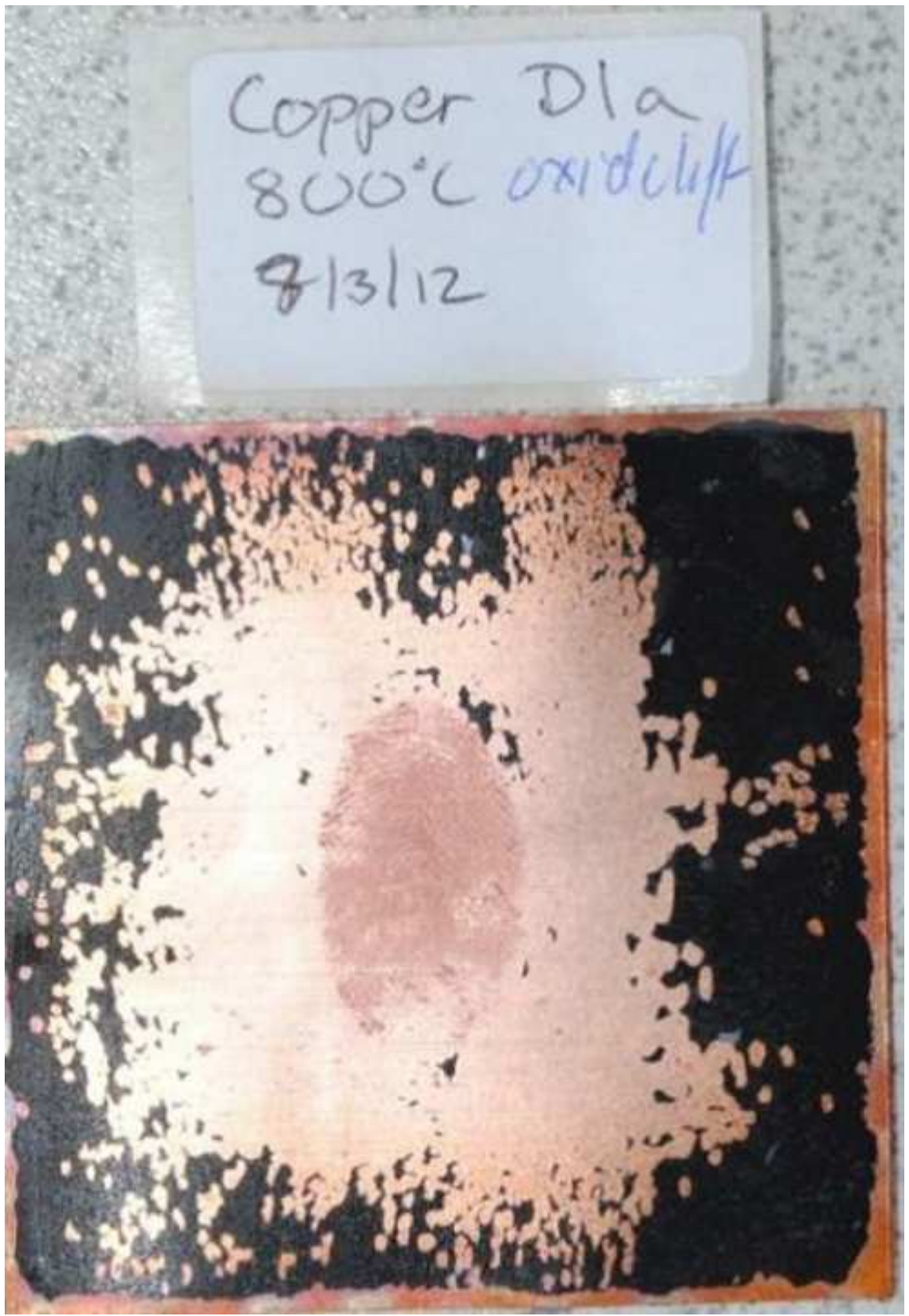



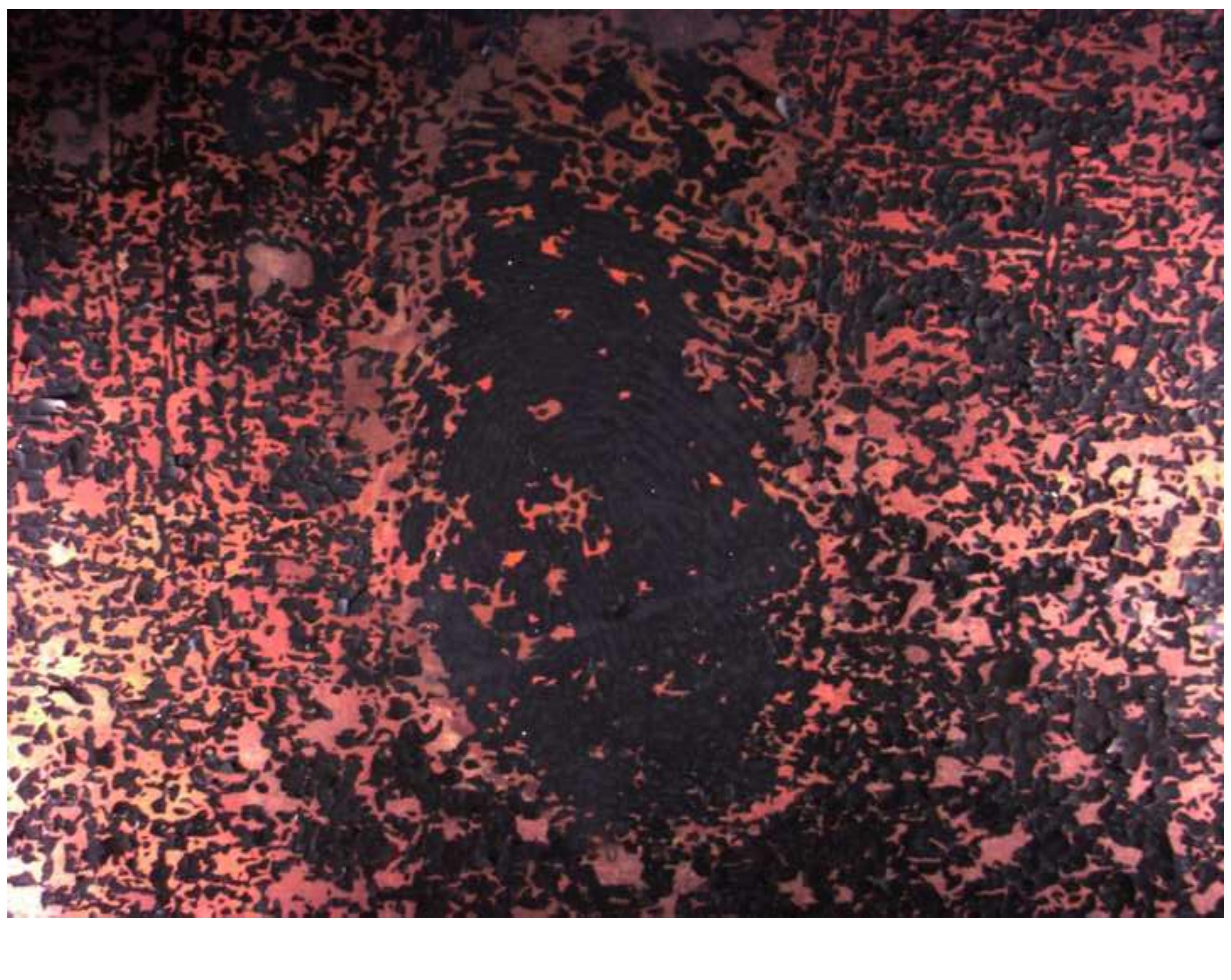
Click here to download high resolution image

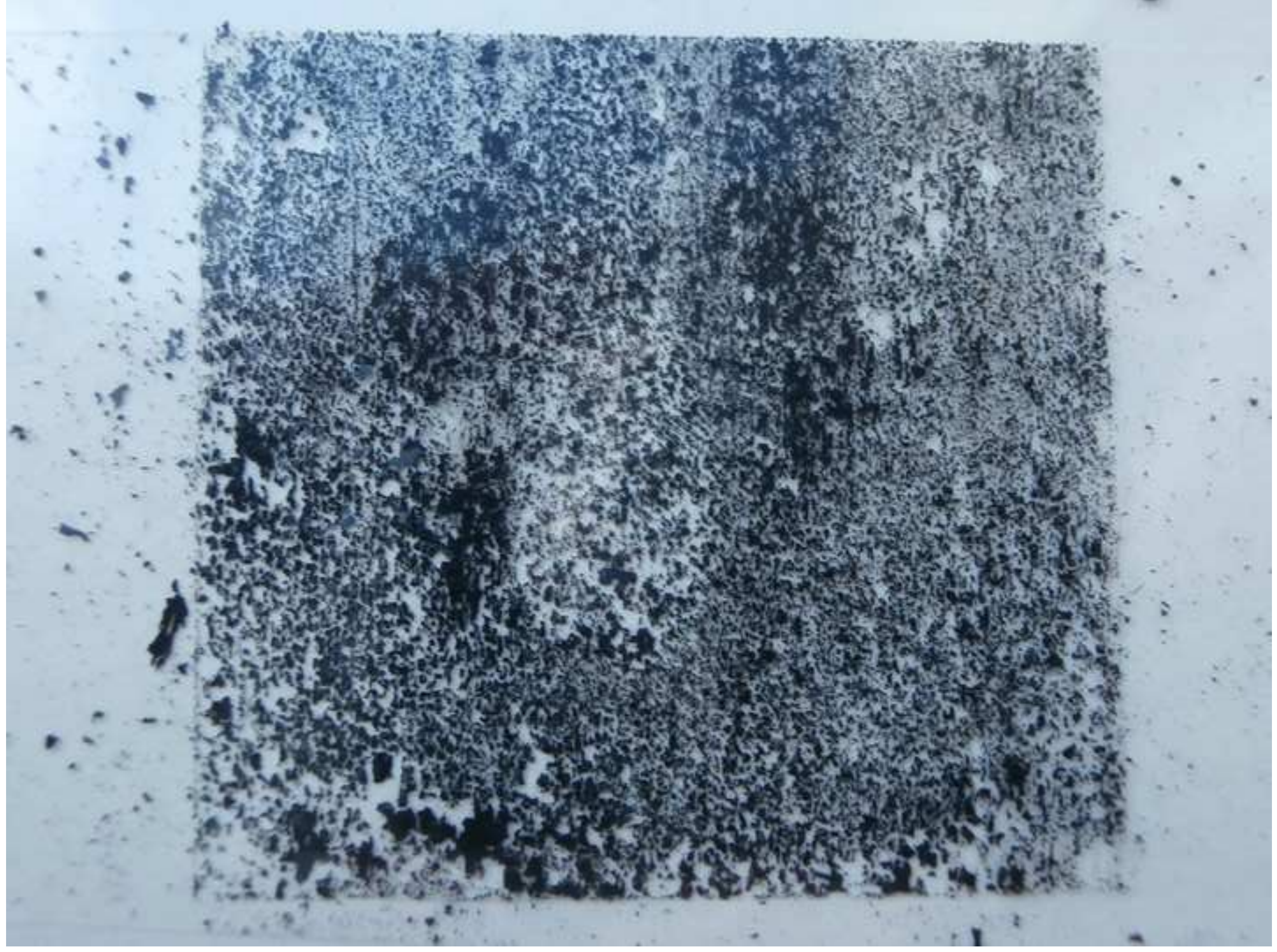


Click here to download high resolution image

Figure 10b

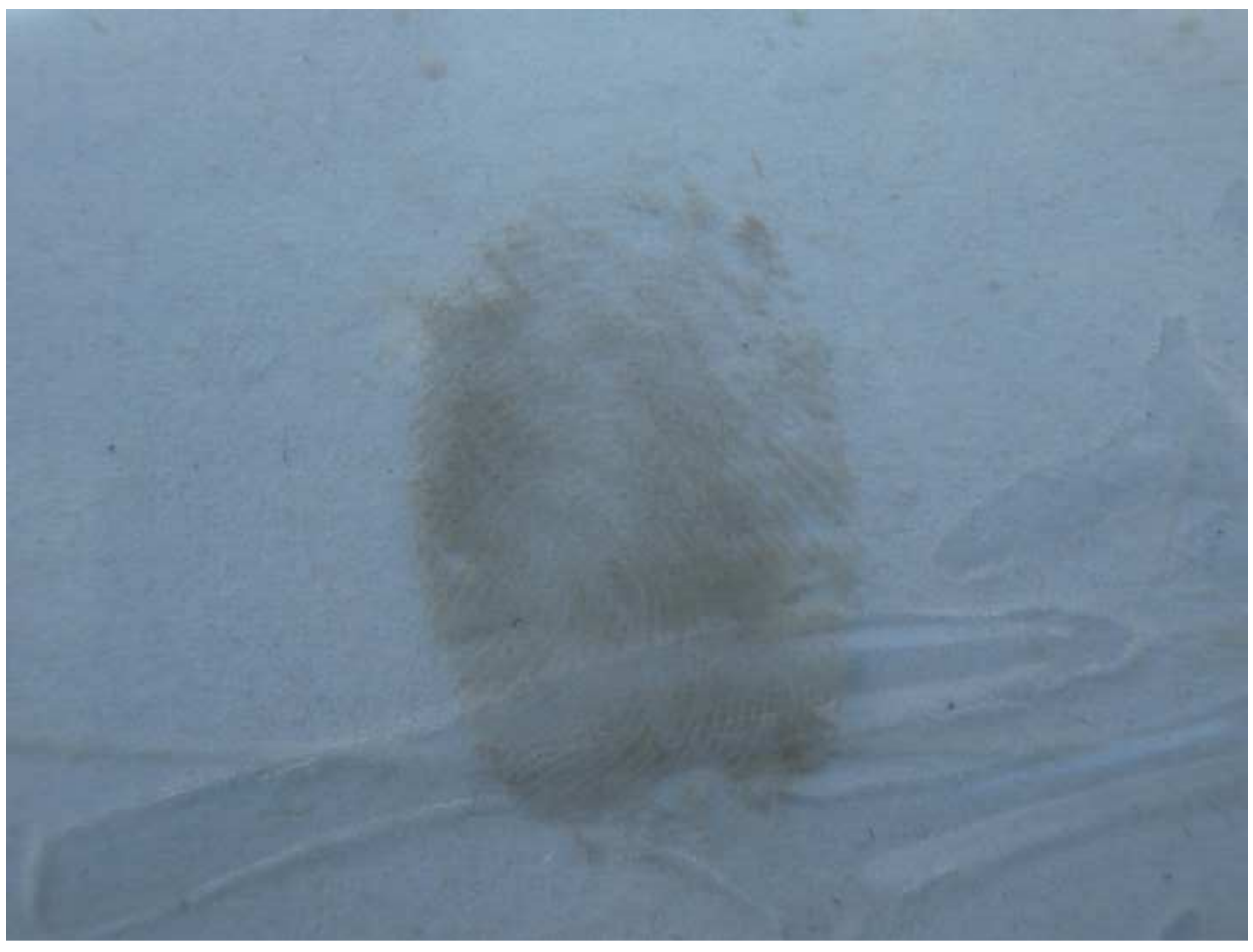


Click here to download high resolution image

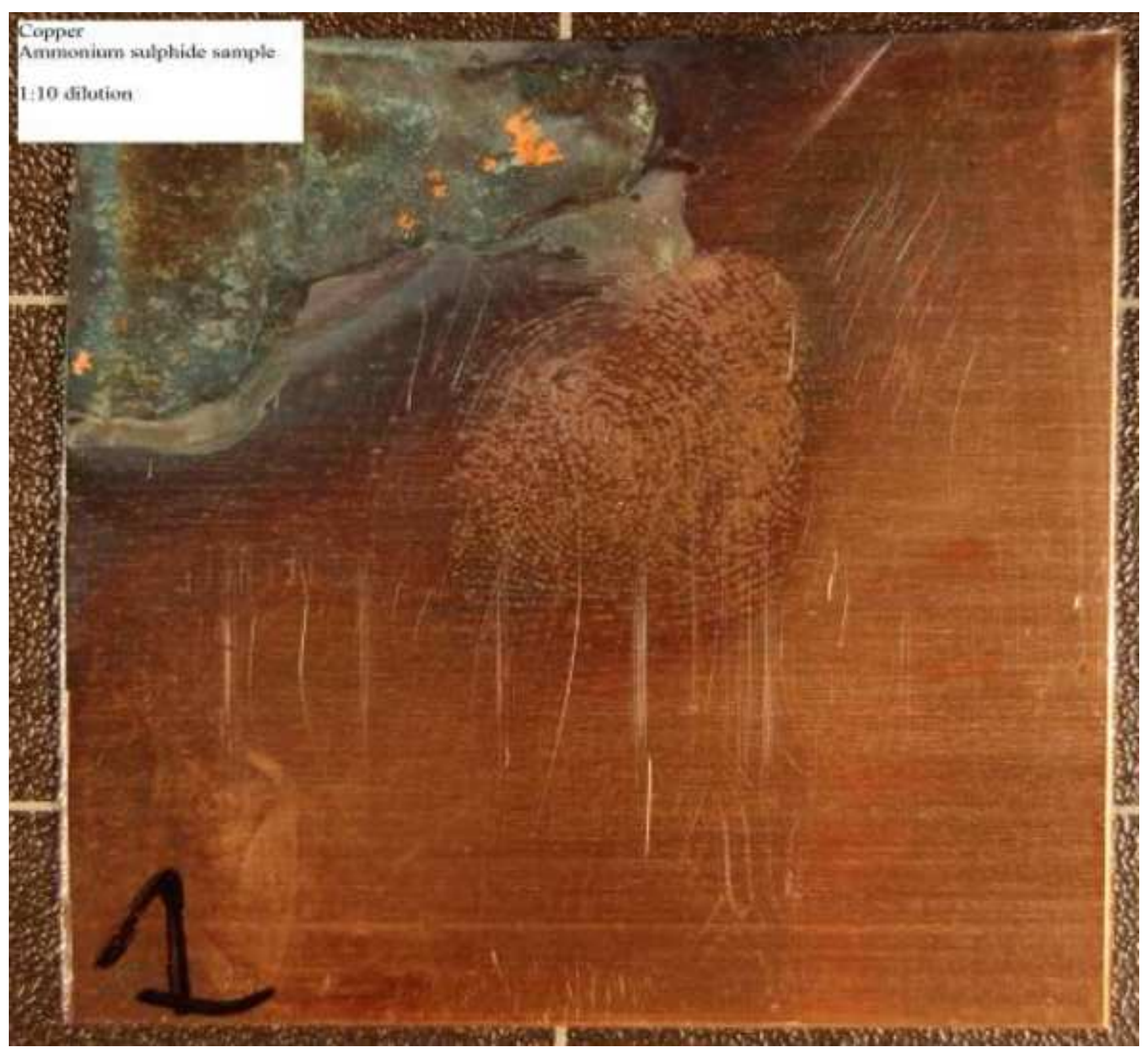




\section{Figure 12a}

Click here to download high resolution image

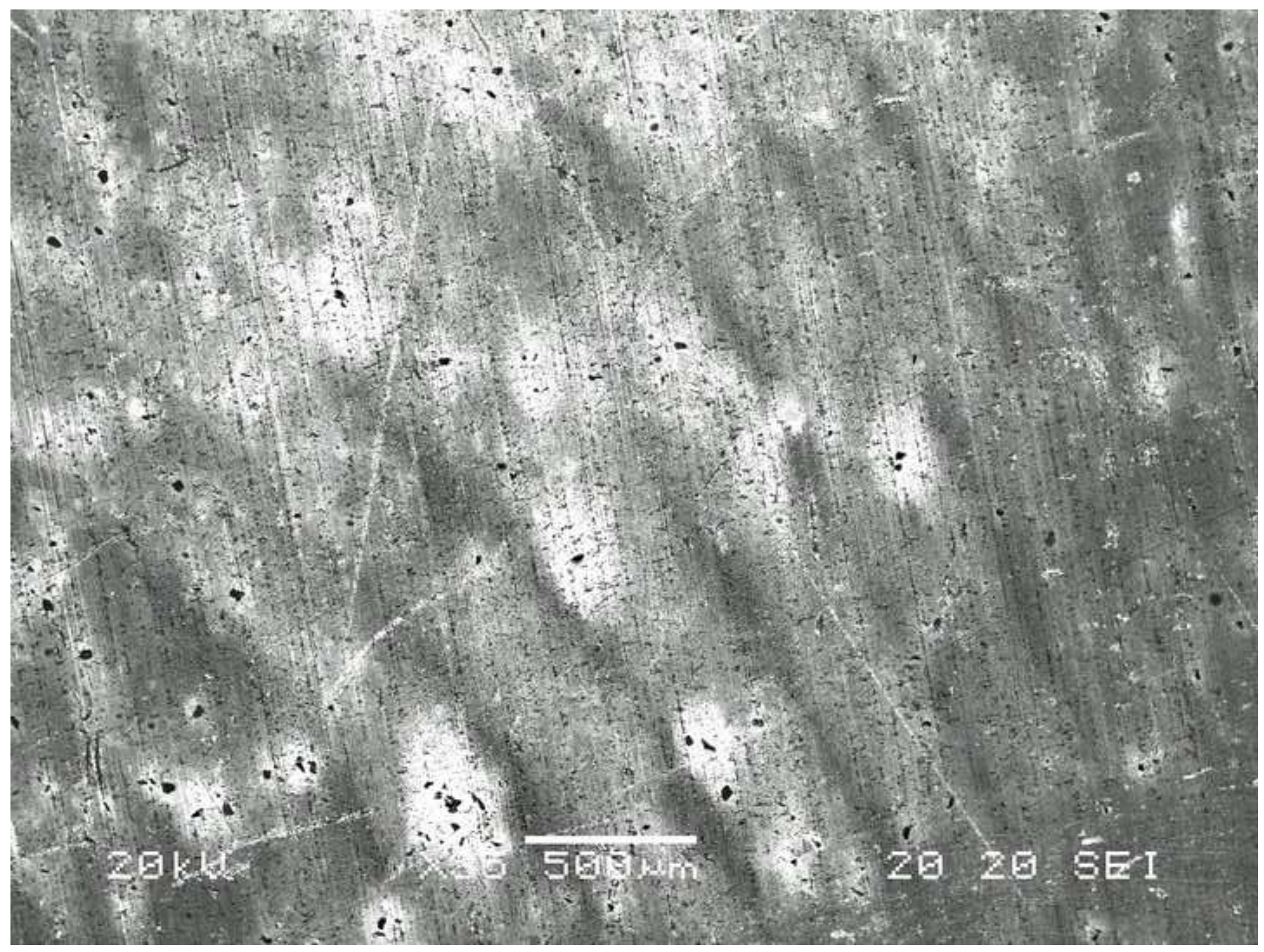


Figure $12 b$

Click here to download high resolution image

If T. 1 h w

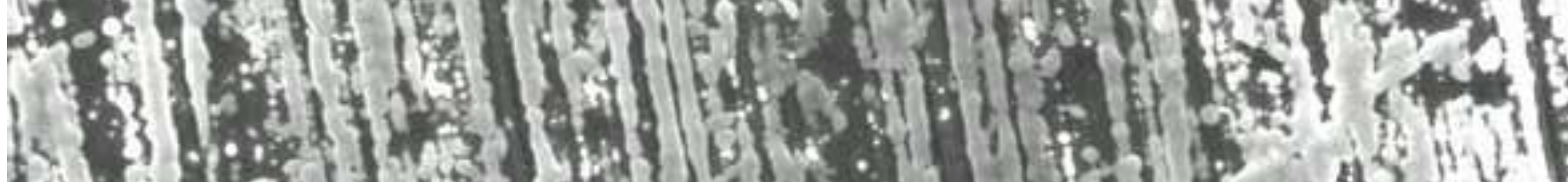

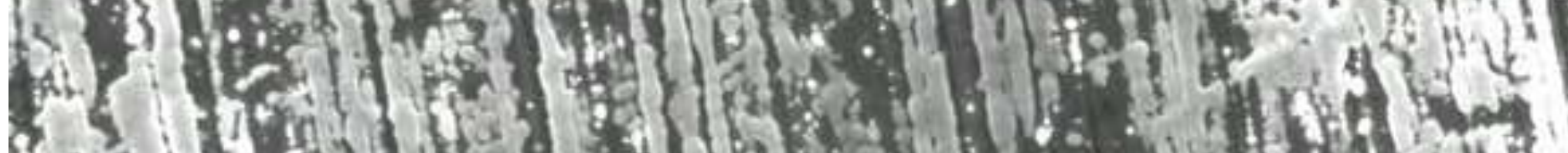
3.

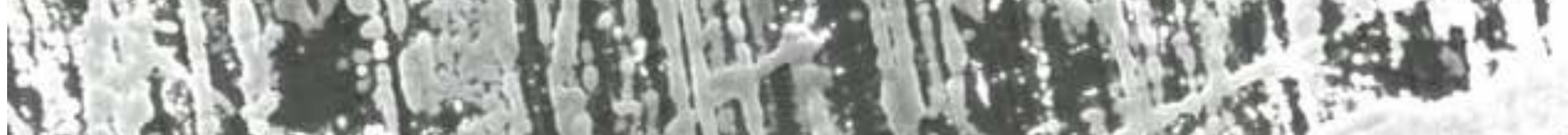
$1+y^{\circ}=$

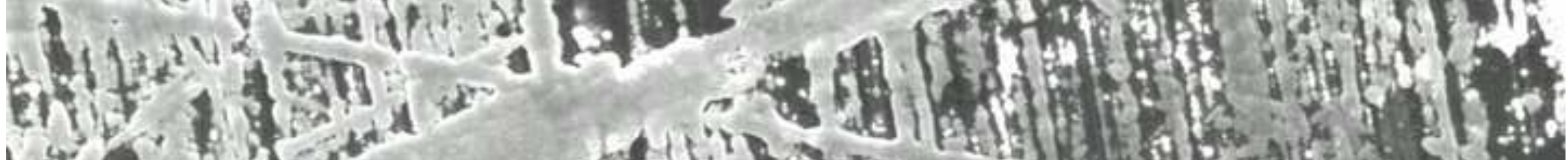
1. (I) 4.

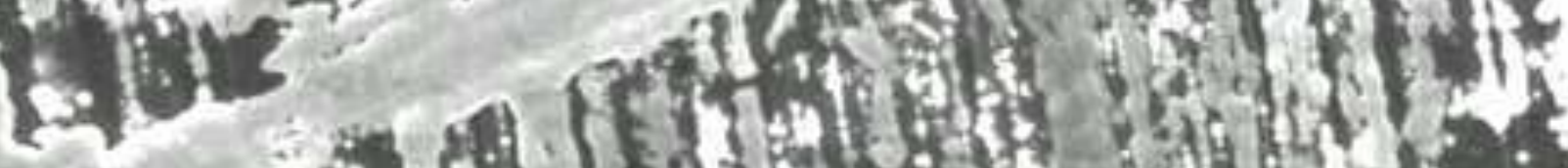

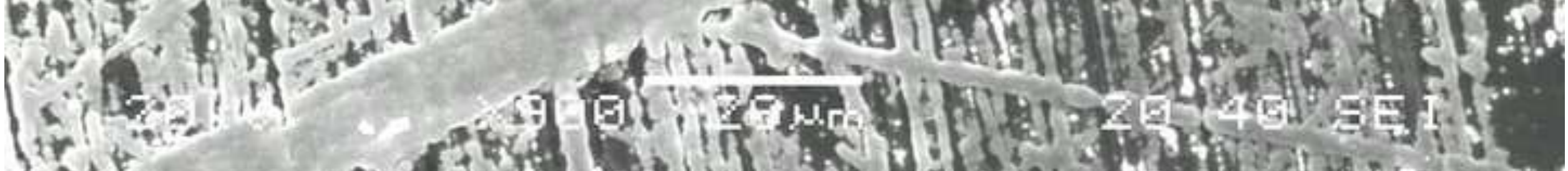
(5) r f

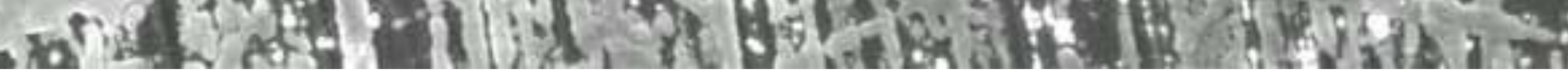




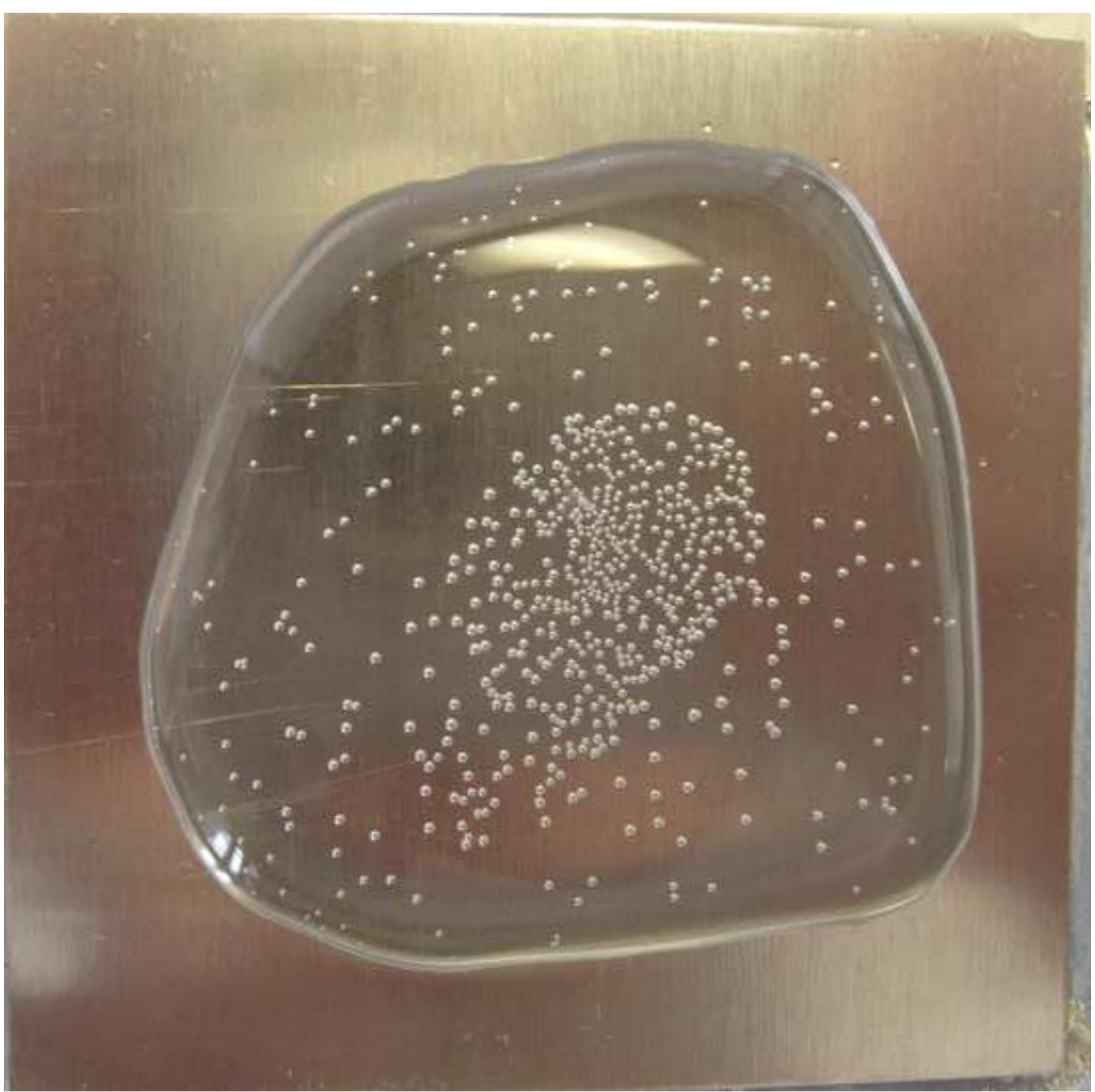




\section{Click here to download high resolution image}

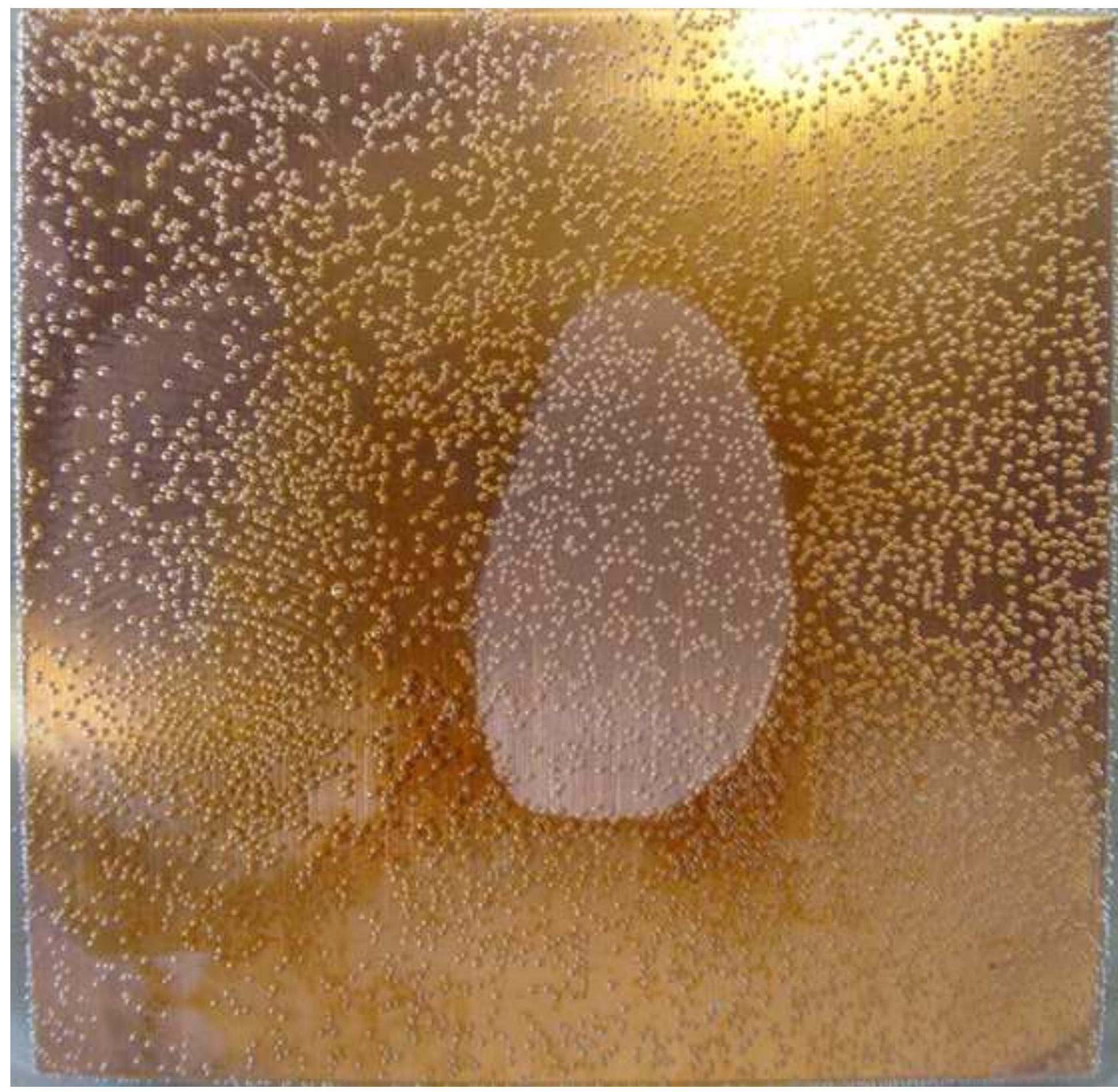




\section{Click here to download high resolution image}

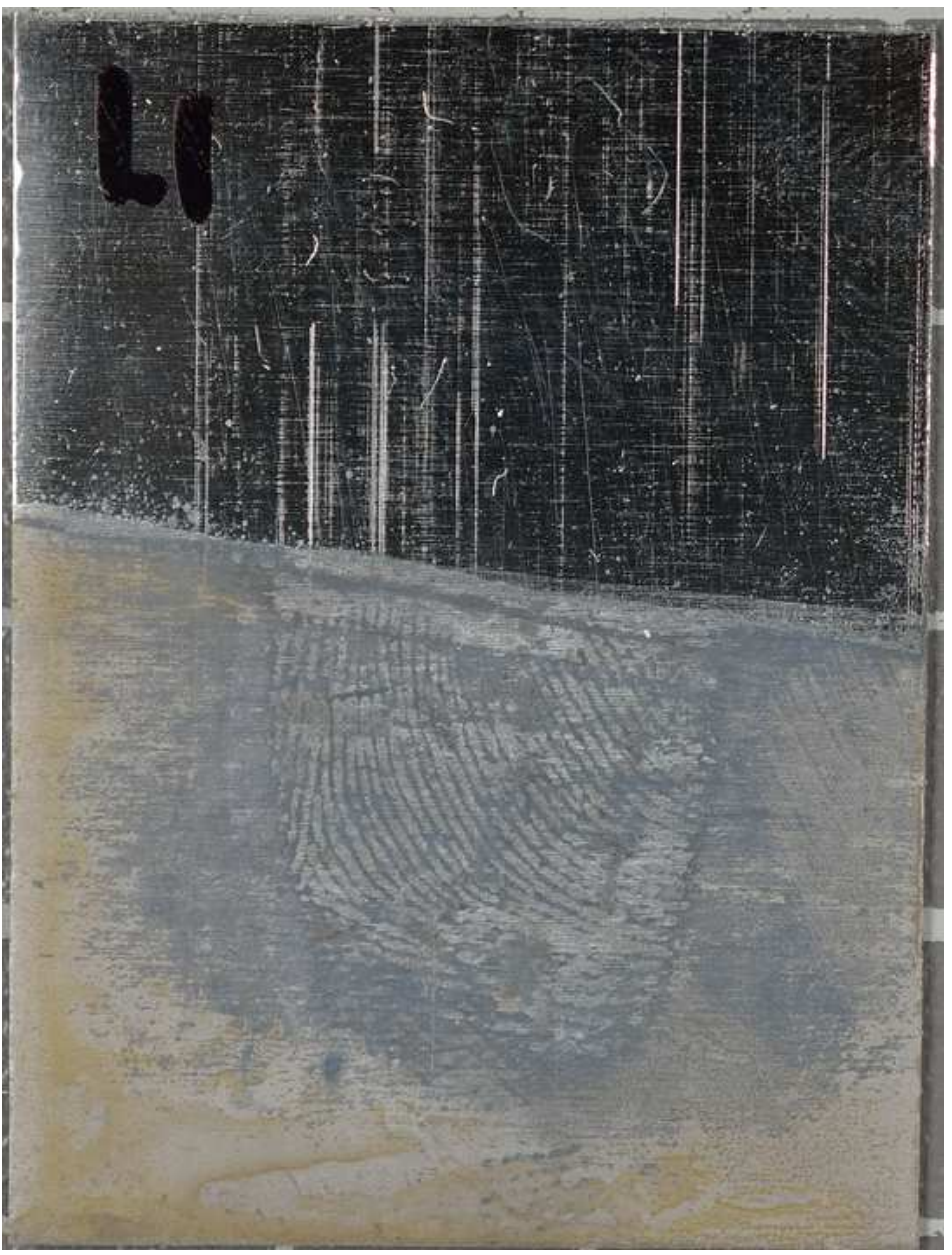




$$
\begin{array}{ll}
1.8 & - \\
&
\end{array}
$$


Click here to download high resolution image

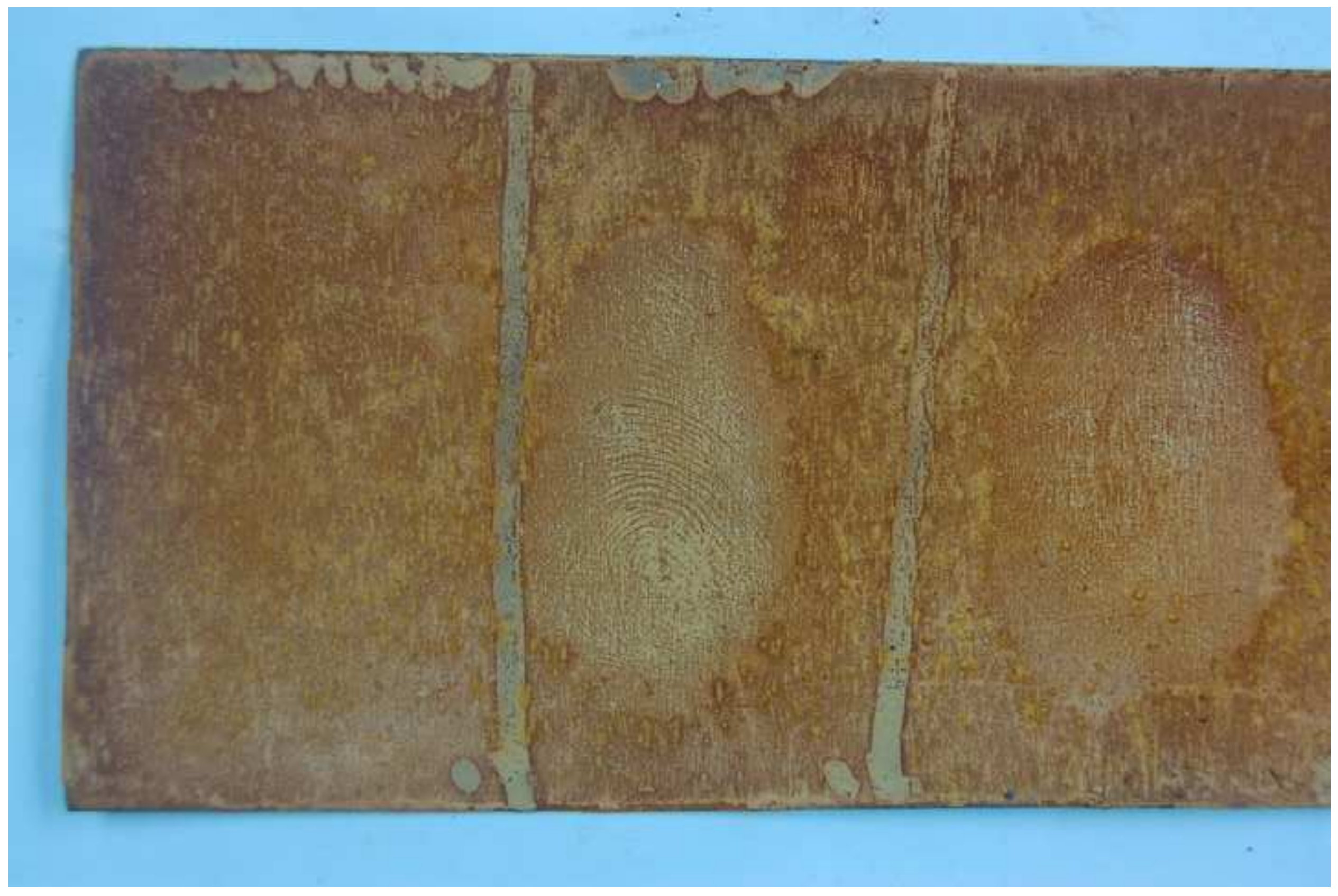




\section{Figure 15b}

Click here to download high resolution image

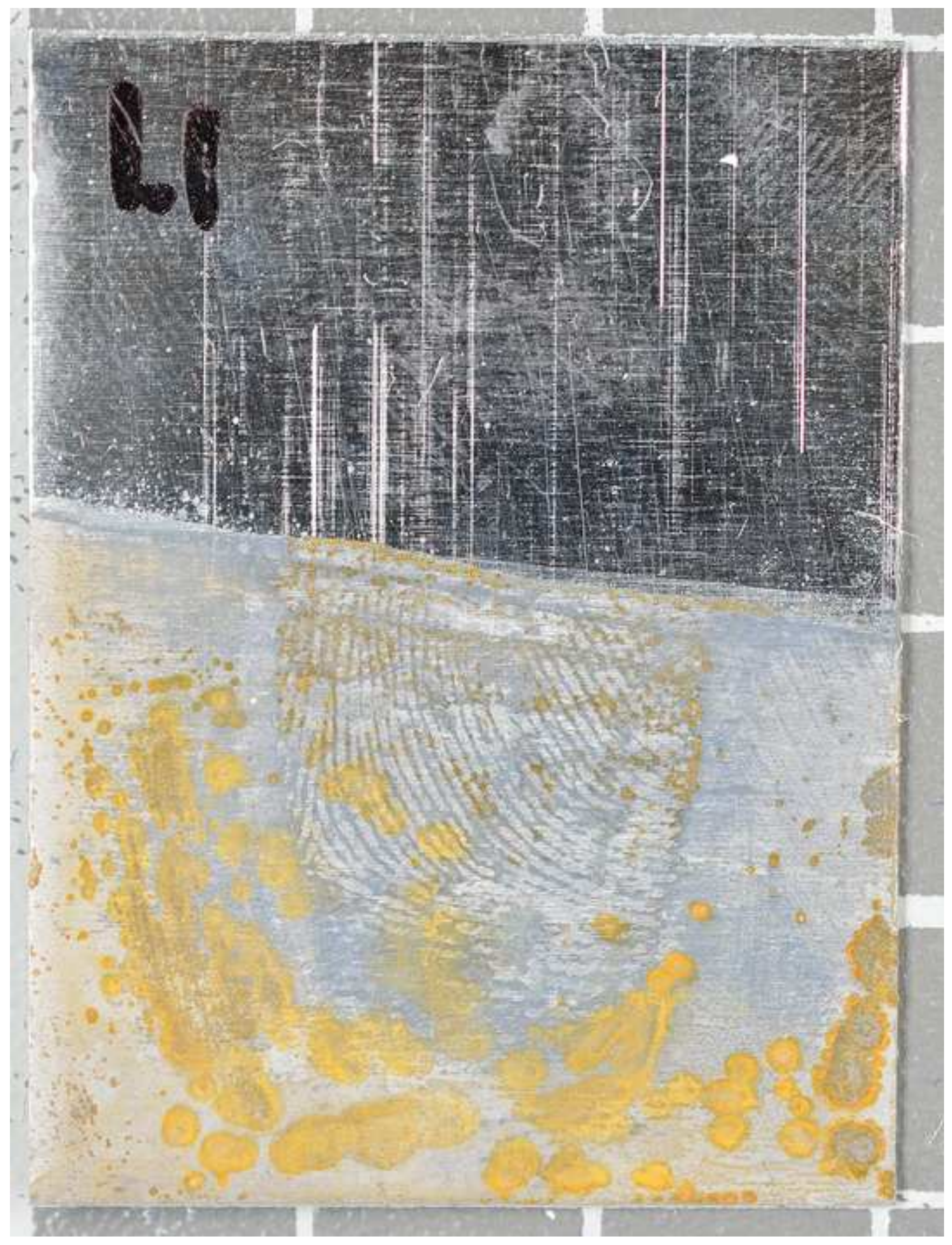

